\title{
Traction forces at the cytokinetic ring regulate cell division and polyploidy in the migrating zebrafish epicardium
}

\author{
Marina Uroz ${ }^{1}$, Anna Garcia-Puig ${ }^{2,3}$, Isil Tekeli ${ }^{2,3}$, Alberto Elosegui-Artola ${ }^{1}$, Juan F. \\ Abenza $^{1}$, Ariadna Marín-Llauradó ${ }^{1}$, Silvia Pujals ${ }^{1}$, Vito Conte ${ }^{1,4}$, Lorenzo Albertazzi ${ }^{1,4}$, \\ Pere Roca-Cusachs ${ }^{1,5}$, Ángel Raya ${ }^{2,3,6}$, and Xavier Trepat ${ }^{1,3,5,6^{*}}$
}

${ }^{I}$ Institute for Bioengineering of Catalonia (IBEC), The Barcelona Institute for Science and Technology (BIST), Barcelona, 08028, Spain

${ }^{2}$ Center of Regenerative Medicine in Barcelona (CMRB), 08003 Barcelona, Spain

${ }^{3}$ Centro de Investigación Biomédica en Red en Bioingeniería, Biomateriales y Nanomedicina (CIBER-BBN), 08028 Barcelona, Spain

${ }^{4}$ Department of Biomedical Engineering and the Institute for Complex Molecular Systems, Eindhoven University of Technology, 5600 MB Eindhoven, The Netherlands

${ }^{5}$ University of Barcelona, 08028 Barcelona, Spain

${ }^{6}$ Institució Catalana de Recerca i Estudis Avançats (ICREA), Barcelona, Spain.

* Corresponding author: xtrepat@ibecbarcelona.eu

Epithelial repair and regeneration are driven by collective cell migration and division. Both cellular functions involve tightly controlled mechanical events, but how physical forces regulate cell division in migrating epithelia is largely unknown. Here we show that cells dividing in the migrating zebrafish epicardium exert large cell-extracellular matrix (ECM) forces during cytokinesis. These forces point towards the division axis and are exerted through focal adhesions that connect the cytokinetic ring to the underlying ECM. When subjected to high loading rates, these cytokinetic focal adhesions (CFAs) prevent closure of the contractile ring, leading to multi-nucleation through cytokinetic failure. By combining a clutch model with experiments on substrates of different rigidity, ECM composition, and ligand density we show that failed cytokinesis is triggered by adhesion reinforcement downstream of increased myosin density. The mechanical interaction between the cytokinetic ring and the ECM thus provides a mechanism for the regulation of cell division and polyploidy that may have implications in regeneration and cancer.

The zebrafish heart has been shown to regenerate a fully functional structure after loss of up to $20 \%$ of the ventricle or genetic ablation of up to $60 \%$ of its cardiomyocyte mass ${ }^{1-3}$. This remarkable regenerative capacity has attracted much interest for its potential to address human cardiovascular disease. Regeneration proceeds through massive proliferation of cardiomyocytes in exquisite coordination with cells from the vascular, nervous and immune systems ${ }^{1,4}$. A particularly relevant tissue in heart regeneration is the epicardium, the mesothelial layer that covers all vertebrate hearts. Following injury, the quiescent zebrafish epicardium re-expresses embryonic genes and secretes soluble factors that regulate cell division, survival and vascularization of new muscle ${ }^{5-8}$.

Two key steps in zebrafish heart regeneration are collective migration and proliferation of epicardial cells to cover the newly synthesized tissue ${ }^{9}$. Both collective cell migration and 
division rely on the generation of physical forces. Physical forces during collective cell migration have extensively been studied using a variety of epithelial and endothelial cell lines ${ }^{10-14}$. By contrast, mechanics of cell division in collective systems remains poorly understood. Here we measured forces at the cell-ECM interface during collective migration and division of the zebrafish epicardium ex vivo. We show that epicardial forces during collective migration differ sharply from previous descriptions in epithelial cell lines. We show, further, that dividing cells connect the cytokinetic ring to the ECM through focal adhesions and generate contractile forces that point towards the division axis. When subjected to high loading rates, these adhesions reinforce and stick the cytokinetic ring to the ECM. This mechanism prevents completion of cytokinesis and gives rise to multinucleated cells.

\section{An unconventional mechanism of collective migration}

To study epicardial forces we dissected hearts from adult zebrafish and gently deposited them on top of collagen-I coated soft polyacrylamide substrates ${ }^{15}$. We chose a substrate stiffness of $14 \mathrm{kPa}$, similar to that of the adult zebrafish heart ${ }^{16}$. A few days after seeding (generally 3-5 days), an epicardial monolayer explanted from the heart and migrated spontaneously on the freely available gel substrate (Fig. 1a, Supplementary Video 1). During migration, cells retained cell-cell adherens junctions rich in $\beta$-catenin (Fig. 1b). Cell and nuclear area were highest at the leading edge and decreased progressively thereafter (Fig. 1c-e). The monolayer was squamous, with a maximum height of $1.3 \pm 0.5 \mu \mathrm{m}$ in the first cell row and $2.3 \pm 0.5 \mu \mathrm{m}$ behind it (mean $\pm \mathrm{SD}$, Supplementary Fig. 1a-e).

The migrating epicardium exhibited a leader/follower organization ${ }^{17}$. Leader cells in the first 2-3 rows showed a dense pattern of stress fibers parallel to the leading edge. These parallel fibers formed at protruding lamellipodia and moved retrogradely towards the cell body (Fig. 1f-g, Supplementary Fig. 2a,b, Supplementary Video 2). Fibers were anchored to the substrate through focal adhesions or connected to neighboring cells through adherens junctions (Fig. 1f,g). Behind the lamellar zone, fibers remained stable and disassembled only when reached by the trailing edge of the cell (Supplementary Video 2). Behind the leading cell rows, basal stress fibers were progressively lost and di-phosphorylation of myosin light chain (ppMLC) decayed (Supplementary Fig. 2,c-d). Immediately above the basal fiber network, we observed a second contractile network lining cell-cell junctions both at the leading edge and behind it (Fig. 1f-h). This cell-cell network was often seen to slide over the more stable basal network, thus indicating that the two contractile networks were largely uncoupled (Supplementary Video 2). To illustrate the relative motion of the two networks we superimposed snap shots of the migrating epicardium of transgenic fish expressing myosin-GFP (Fig. 1h). Maps color-coding myosin as a function of time revealed that basal fibers were mostly immobile as the apical network moved over them. Despite their strikingly distinct cytoskeletal organization, leaders and followers were not different cell types, as shown by the fact that follower cells quickly begun to assemble stress fibers upon spontaneous death of single followers (Supplementary Fig. 2e, Supplementary Video 3 ). Moreover, staining of tcf-21 confirmed that all cells in the monolayer were epicardial (Fig. 1b). 
The pattern of basal stress fibers parallel to the leading edge differs from previous studies in expanding monolayers ${ }^{10,18}$, suggesting a new mechanism for force generation during collective cell migration. To study this mechanism, we used traction force microscopy (TFM) to map forces at the cell-ECM interface (Fig. 2a-c, Supplementary Video 4) ${ }^{13}$. As expected from myosin organization, traction forces in the first cell row were mainly parallel to the leading edge and dropped quickly thereafter (Fig. 2a-b). High resolution force maps revealed that force transmission to the ECM was localized at the sites of stress fiber anchoring (Fig. 2c). This behavior is reminiscent of closure of small wounds, during which the attachment of a supracellular actomyosin cable to the substrate generates large parallel tractions ${ }^{19}$. However, it contrasts sharply with previous studies in expanding epithelial and endothelial monolayers, which showed that cells exert the largest forces towards the empty space independently of cell motion and geometry ${ }^{13,14,20}$. Our data show that the zebrafish epicardium uses an unconventional mechanism of collective migration in which cells contract the substrate parallel to the direction of motion, rather than perpendicular to it.

We studied whether this force pattern can explain the distribution of cell areas during migration. Despite the fact that tangential tractions were dominant in magnitude, their integration over the field of view vanished (Fig. 2b, inset). Therefore, their net effect on global migration can be neglected in a first approximation. By contrast, we measured a net inward-pointing radial traction at the leading edge. Since traction forces add up to zero in a vectorial sense when summed over the cell sheet, this local net radial traction indicates that leader cells pull on their followers. To study how leader forces impact follower migration and deformation we adapted a 1D model of a migrating monolayer ${ }^{10}$. The model treats the monolayer as a series of springs connected in series (Fig. 2d). Each spring mimics a cell and each connection between them mimics a cell-cell junction. Cell-ECM interaction is modelled by viscous elements that connect each junction with the underlying substrate. The monolayer is pulled forward by a self-propelling force generated by the leading cell. This pulling force is balanced by the elastic force at the springs and by the viscous force at the cell-substrate interface (Fig. 2e). Following experimental observations during early explantation, all cells in the initial configuration of the modeled monolayer are chosen to have the same size (Fig. 2f). The onset of force generation at the leading edge then begins to stretch the uniform monolayer. If cell-substrate friction was negligible, force generated at the leading edge would be immediately transmitted to all cells in the monolayer, and the distribution of cell size would remain uniform. However, non-negligible viscous friction with the substrate prevents instantaneous transmission of the leading force to the passive followers. As a consequence, the further a cell is from the leading edge, the smaller is its deformation, which results in a gradient of cell size similar to that observed experimentally (Fig. 2g). Thus, a leader-follower organization is sufficient to explain the observed gradient in cell area.

\section{Epicardial cells exert traction forces during cytokinesis}

We next focused on mechanics of cell division during epicardial expansion. Current understanding emphasizes that nearly all eukaryotic cells lacking a cell wall round up during mitosis ${ }^{21,22}$. In contrast with this view, epicardial cells remained flat and maintained a constant eccentricity throughout division (Fig. 3a-c, Supplementary Video5). At the onset 
of metaphase, traction forces under the dividing cell decreased, consistent with the prevalent notion that cells inactivate integrins and weaken adhesion with the ECM to divide (Fig. 3d,e) 23-25. During cytokinesis traction forces increased sharply (Fig. 3d,e). Forces were restricted to the interface between the two daughter cells and pointed towards the division axis as force dipoles. These dipoles appeared shortly after the accumulation of myosin at the cleavage furrow (Fig. 3f, Supplementary Video 6), suggesting that the cytokinetic ring is not isolated from its inert surroundings but rather dynamically anchored to the ECM. To assess this possibility, we studied the localization of paxillin, using both immunostainings of phopho-paxillin (p-paxillin) and live imaging of a line expressing paxillin-GFP'26, 27 (Fig. 3g,h, Supplementary Video 7). At the onset of metaphase paxillin was distributed homogeneously in the cytoplasm. When the contractile ring formed, large focal adhesions appeared on each side of ventral stress fibers under the cleavage furrow, indicating that the contractile machinery that drives division is coupled to the ECM (Fig. $3 \mathrm{~g}, \mathrm{~h})$. STORM imaging revealed a dense F-actin network between focal adhesions at the basal plane of the cytokinetic ring (Fig. 3i). To distinguish these adhesions from focal adhesions at the cell periphery we call them CFAs. As the cytokinetic ring contracted, CFAs moved inwards, and they disappeared when the ring disassembled (Supplementary Video 7).

\section{Reinforcement of CFAs leads to cytokinetic failure}

A remarkable feature of epicardial regeneration in zebrafish is the recent discovery that cells close to the leading edge are multinucleated both in vivo and ex vivo ${ }^{17}$ (Fig. 4a-d). The fraction of multinucleated cells was highest at the leading edge, where half of the cells exhibited more than one nucleus (Fig. 4b). Multinucleation is characteristic feature of a variety of cell types in physiology and disease ${ }^{28,29}$, and it is usually attributed either to cell fusion $^{30}$ or failed division ${ }^{31}$. We did not observe fusion events, but cells were often seen to fail cytokinesis (Fig. 4e, Supplementary Video 8). As in successful divisions, the contractile ring of cells failing cytokinesis was anchored to the ECM through CFAs (Fig. 4e,f). After 60 minutes, well after successful divisions were completed, CFAs disassembled and the furrow regressed without reaching abscission, thereby giving rise to a binucleated cell (Fig. 4e,f, Supplementary Video 9).

Failed cytokinesis is a hallmark of diverse processes in morphogenesis and disease, such as hepatocyte differentiation and aberrant division of cancer cells $\mathrm{s}^{31-33}$. In these processes, cytokinetic failure is often caused by insufficient contractility of the ring, which prevents ingression of the cleavage furrow. To test whether this mechanism explained our observations in the zebrafish epicardium, we compared myosin intensity in cells exhibiting successful and failed cytokinesis (Fig. 5a). Contrary to our expectations, myosin accumulation at the cytokinetic ring was higher in cells failing cytokinesis, thus ruling out insufficient contractility as the mechanism underlying cytokinetic failure. This observation led us to focus on mechanosensing at CFAs in response to ring contraction. We reasoned that, rather than favoring contraction, excess myosin could trigger reinforcement feedback loops ${ }^{34,35}$ at the cell-ECM contacts under the ring, thereby generating long-lived CFAs. In this hypothetical scenario, excess force would favor adhesion of the ring to the ECM and prevent cytokinesis from being completed. 
To test this hypothesis, we turned to a clutch model of cell adhesion, which has been shown to explain cellular sensing of rigidity and ECM ligand density ${ }^{35-38}$ (Fig. 5c-e). We modeled the basal side of the contractile ring as a 1D actin filament pulled by myosin motors and anchored to the substrate through a parallel arrangement of molecular clutches. Each clutch comprises one integrin and a mechanosensing adaptor protein. Actin is pulled centripetally by myosin motors in the ring according to a linearly decreasing force-velocity relationship. Myosin force generates an actin retrograde flow which progressively pulls attached clutches towards the center of the cytokinetic ring. Bond dynamics of the clutches is modeled using a "weakest link" approach, which assumes that the integrin-ECM bond, the adaptor-integrin bond, and the adaptor-actin bond can simply be modeled using one set of binding (kon) and unbinding (koff) rates corresponding to the weakest of these bonds. When force transmitted to each clutch reaches a threshold value, a reinforcement mechanosensing event is triggered, leading to an increase in integrin number. In this interpretation of focal adhesions in terms of molecular clutches, the key variable that determines whether an adhesion will fail or reinforce is the loading rate that the actomyosin cytoskeleton applies to each clutch. When the loading rate is low, force leads to clutch unbinding from the matrix before the reinforcement threshold is reached, and the actomyosin ring slips centripetally. When the loading rate is high, by contrast, the reinforcement threshold is reached before clutches unbind, leading to growth of focal adhesions and immobilization of the actomyosin ring on the substrate.

We then used the model to predict the time evolution of traction forces and ring closure velocity, which is taken simply as the magnitude of the actin retrograde flow. To this end, we fed the model with the experimentally measured time evolution of myosin accumulation in successful and failed cytokinesis events (Fig. 5a). Model predictions depend critically on the magnitude of the reinforcement threshold. When this threshold is high, then the model predicts that mechanosensing feedback loops are never triggered and the ring that contains higher amount of myosin closes faster and generates lower traction forces (Supplementary Fig. 3a,b). Conversely, when the reinforcement threshold is low, then mechanosensing feedback loops are readily triggered, large focal adhesions grow, and the rings never close, irrespective of the amount of myosin (Supplementary Fig. 3e,f). None of these scenarios is consistent with our observation that rings with higher myosin density fail to close. At intermediate values of the reinforcement threshold, however, the model predicts a regime in which more reinforcement events are triggered for higher myosin densities. As a consequence, the ring with higher myosin density closes slower and exerts higher traction forces than the ring with lower myosin density (Fig. 5f, Supplementary Fig. 3c,d). To test whether this intermediate scenario is consistent with our observations, we measured traction forces exerted by the cytokinetic ring during successful and failed cytokinesis. Consistent with model predictions (Fig. 5f), cells undergoing cytokinetic failure exerted traction forces and loading rates that were more than 3-fold higher than those that succeeded cytokinesis (Fig. 5g,1). In addition, rings generating the highest tractions closed at the slowest velocity (Fig. 5h). Similar results were obtained on substrates coated with fibronectin rather than collagen (Supplementary Fig. 4).

We also studied cytokinesis dynamics on substrates of lower rigidity $(5 \mathrm{kPa})$. The fraction of failed cytokinesis events was not significantly different between $5 \mathrm{kPa}$ and $14 \mathrm{kPa}$ $(21 \pm 7 \%$ and $25 \pm 6 \%$, respectively, mean \pm SEM). The time evolution of traction forces 
generated by cells succeeding cytokinesis was qualitatively similar on soft and stiff substrates, but with quantitative differences. Like cells on stiff substrates, cells that failed cytokinesis on soft substrates exhibited higher myosin density at the ring (Fig. 5b) and, as predicted by our model (Fig. 5i), higher traction forces (Fig. 5j). Also consistent with our model, cytokinetic failure on soft substrates was associated with higher loading rates and slower closure velocities (Fig. 5k,1). A final feature of focal adhesions that is well captured by clutch models is that, for substrates of sufficiently high rigidity, focal adhesions grow in size with increasing ECM ligand density ${ }^{36}$. We thus hypothesized that increasing ECM ligand density should increase CFA size and limit successful cytokinesis. To test this hypothesis, we placed hearts on micropatterned glass substrates exhibiting parallel stripes of high and low fibronectin density (Fig. 5m,o). Cells migrating on the areas of high density exhibited larger focal adhesions (Fig. 5o). As predicted, we observed that failed divisions were more frequent in the areas of high fibronectin density (Fig. 5n). Together, these results rule out that cytokinesis fails because of a lack of contractile force at the cytokinetic ring. On the contrary, high myosin levels trigger adhesion reinforcement feedback loops, which prevents cells from disengaging adhesions between the cytokinetic ring and the ECM.

\section{Discussion}

Physical forces driving collective cell migration have extensively been documented in a variety of epithelial and endothelial monolayers ${ }^{10-14,39}$. Whether and how traction forces impact division in cell collectives has been largely unexplored, however. To a great extent, this gap of knowledge arises from the fact that dividing cells weaken their adhesion with the substrate to round up and they re-spread only after cytokinesis is complete ${ }^{21-25}$. As a consequence, the dividing cell does not generate significant tractions during mitosis and cytokinesis $^{40,41}$. Here we found that cells in epicardial explants detach from the matrix to divide but they do not round up. Rather, they remain squamous and strongly adhered to their neighbors. At the onset of cytokinesis, the contractile ring adheres to the underlying matrix and generates large traction forces. Thus, in order to succeed at cytokinesis, the dividing cell must overcome friction originating from specific adhesion with the matrix.

We identified that transient adhesion of the cytokinetic ring with the ECM is mediated by CFAs. Integrin dynamics has long been involved in mitosis and cytokinesis ${ }^{41-48}$ but, even at the single cell level, little is known about force generation and adhesion with the ECM during cell division. Before the development of traction microscopy, Burton and Taylor provided evidence that, during cytokinesis, single isolated fibroblasts were able to wrinkle the thin elastic membrane on which they were adherent, indicating force transmission between the cell and its substrate ${ }^{49}$. By contrast, dictyostelium cells and isolated MDCK cells probed with TFM did not exert forces during cytokinesis ${ }^{40,50}$. In confluent monolayers, we recently showed that MDCK cells round up for division and do not exert tractions during cytokinesis ${ }^{40}$. This is in sharp contrast with epicardial monolayers studied here, which did not round up for division and exerted tractions during cytokinesis. These differences between MDCK cells and zebrafish epicardial cells might arise from the very different geometry of the two cell types. MDCK cells are cuboidal whereas epicardial cells are squamous. A cuboidal cell in a monolayer will easily round up and divide without severely compromising the integrity of the monolayer. By contrast, a flat cell would require 
pronounced changes in the monolayer geometry to round up for division, including deformation of the neighbors in the three dimensions. During cytokinesis, MDCK cells are round and not in contact with the substrate, so it is straightforward to conclude that they will not be able to generate tractions. By contrast, the basal surface of epicardial cells remains in contact with the substrate throughout division, favoring adhesion between the ring and the ECM coating the substrate.

We found that a fraction of migrating epicardial cells was polyploid, both in vivo and in vitro $^{17}$. Polyploidy is widely seen as negative hallmark of cancer and aging ${ }^{31}$, but it is also a physiological feature of cell types with high regenerative capacity. Physiological examples of multinucleated cells includes trophoblast giant cells in the placenta, hepatocytes in the liver, megakaryocytes in the bone marrow, osteoclasts in the bone, and myocytes in skeletal muscle $^{28}$. Previous studies proposed a role for polyploid cells in wound healing and cell migration. For example, polyploid cells were reported in the granulation tissue of wellhealing human wounds, whereas they were absent in chronic wounds ${ }^{51}$. In a later study, polyploidy was shown to drive wound healing following puncture wounds in the drosophila epidermis $^{29}$. Recently, Cao et al showed a large fraction of cells at the leading edge of the regenerating zebrafish epicardium is polynucleated ${ }^{17}$. Potential mechanisms by which polyploid cells might display enhanced regenerative capacity are largely unknown, but they might include increased cell size, minimization of membrane requirement through decreased surface-to-volume ratio, increased metabolic capacity, the generation of genetic variability, and rapid protein synthesis and enhanced protrusion and migration ${ }^{28,52}$. Further studies should address whether the mechanisms of polyploidy identified here favor the outstanding regenerative capacity of the zebrafish epicardium.

Multinucleated cells can be generated either by cell fusion ${ }^{30}$, which we did not observe here, or by failed cytokinesis. Cytokinesis may fail at several stages due to inactivation or hyperactivation of any of its multiple components. Existing mechanisms include precocious or delayed ingression due to enhanced of impaired contractility of the actomyosin ring, destabilization of the microtubule machinery, or inhibition of abscission ${ }^{53}$. In the zebrafish epicardium, failed cytokinesis was recently associated with increased tension, but the mechanism by which tension might cause cytokinetic failure was unknown ${ }^{17}$. Here we showed that the mechanism by which cytokinesis fails in the zebrafish epicardium is determined by the interaction between the cytokinetic ring and the ECM. We found that cells failing cytokinesis displayed higher myosin accumulation. This result might seem counterintuitive as increased myosin at the ring could be expected to enhance its contractility rather than impede it. A regime in which higher accumulation of myosin II is paralleled by slower ring closure has been previously identified and attributed to crosslinking ${ }^{54,55}$. Potential underlying mechanisms include stiffening of the cytoskeletal cortex by stabilization of filament interactions, reduction in cortical dynamics by suppression of actin depolymerization, and increasing of steric hindrance. Here, by contrast, we found that the ring contractility was slowed down by friction with the substrate at CFAs. In parallel with increased myosin we observed higher tractions and higher loading rates. Supported by a theoretical description of cell adhesion in terms of molecular clutches, our results indicate that increased myosin triggers feedback loops that reinforce focal adhesions and prevent the ring from sliding centripetally. The interaction between the 
cytokinetic ring and focal adhesions thus provides a new mechanism for the generation of multinucleated polyploid cells, with potential implications in physiology and disease.

The rate of polyploidy was highest at the leading edge and decreased away from it. This gradient in polyploidy coincided with the gradient in cell area, which suggests a mechanistic link. One possible underlying mechanism is provided by differential myosin activity as a function of cell area. Cell area has long been associated with increased phosphorylation of the MLC through mechanisms that remain poorly understood ${ }^{56}$. In the zebrafish epicardium, cell area and phosphorylation of the MLC are also strongly correlated (Supplementary Fig. 2c-d) ${ }^{17}$. In light of these results, we propose the following biophysical picture to explain the distribution of polyploidy in the monolayer. First, the leader-follower organization generates a gradient of cell area, as supported by our physical model. This gradient results in a gradient of phosphorylated MLC. Cells with higher MLC phosphorylation then display higher contractility of their cytokinetic ring, which favors cytokinesis failure through reinforcement of CFAs. This picture, which should be further evaluated in future studies, couples migration forces with division failure.

In summary, we showed that epicardial cells dividing in a collective generate forces on their extracellular matrix. These forces are restricted to cytokinesis and originate from a connection between the contractile ring and the ECM mediated by paxillin-rich focal adhesions. During normal cytokinesis, force loading of these adhesions is slow and enables the ring to overcome matrix adhesion. However, higher forces cause adhesion reinforcement, which impedes sliding of the ring and leads to cytokinetic failure and multinucleation. Thus, our results establish a direct mechanism by which adhesion with the ECM controls cytokinesis.

\section{References}

1. Jopling, C. et al. Zebrafish heart regeneration occurs by cardiomyocyte dedifferentiation and proliferation. Nature 464, 606-609 (2010).

2. Raya, Á. et al. Activation of Notch signaling pathway precedes heart regeneration in zebrafish. Proc. Natl. Acad. Sci. USA 100, 11889-11895 (2003).

3. Wang, J. et al. The regenerative capacity of zebrafish reverses cardiac failure caused by genetic cardiomyocyte depletion. Development 138, 3421-3430 (2011).

4. Lien, C.-L., Harrison, M.R., Tuan, T.-L. \& Starnes, V.A. Heart repair and regeneration: Recent insights from zebrafish studies. Wound Repair Regen. 20, 638646 (2012).

5. Lepilina, A. et al. A Dynamic Epicardial Injury Response Supports Progenitor Cell Activity during Zebrafish Heart Regeneration. Cell 127, 607-619 (2006).

6. Wei, K. et al. Epicardial FSTL1 reconstitution regenerates the adult mammalian heart. Nature 525, 479 (2015).

7. Huang, G.N. et al. C/EBP Transcription Factors Mediate Epicardial Activation During Heart Development and Injury. Science 338, 1599-1603 (2012).

8. Peralta, M., González-Rosa, J.M., Marques, I.J. \& Mercader, N. The Epicardium in the Embryonic and Adult Zebrafish. J. Dev. Biol. 2, 101-116 (2014).

9. Wang, J., Cao, J., Dickson, A.L. \& Poss, K.D. Epicardial regeneration is guided by cardiac outflow tract and Hedgehog signalling. Nature 522, 226-230 (2015). 
10. Serra-Picamal, X. et al. Mechanical waves during tissue expansion. Nat. Phys. 8, 628-634 (2012).

11. Reffay, M. et al. Interplay of RhoA and mechanical forces in collective cell migration driven by leader cells. Nat. Cell Biol. 16, 217 (2014).

12. Das, T. et al. A molecular mechanotransduction pathway regulates collective migration of epithelial cells. Nat. Cell Biol. 17, 276-287 (2015).

13. Trepat, X. et al. Physical forces during collective cell migration. Nat. Phys. 5, 426430 (2009).

14. du Roure, O. et al. Force mapping in epithelial cell migration. Proc. Natl. Acad. Sci. USA 102, 2390-2395 (2005).

15. Kim, J., Rubin, N., Huang, Y., Tuan, T.-L. \& Lien, C.-L. In vitro culture of epicardial cells from adult zebrafish heart on a fibrin matrix. Nat. Protoc. 7, 247255 (2012).

16. Garcia-Puig, A. et al. Proteomics analysis of extracellular matrix remodeling during zebrafish heart regeneration. bioRxiv, 588251 (2019).

17. Cao, J. et al. Tension Creates an Endoreplication Wavefront that Leads Regeneration of Epicardial Tissue. Dev. Cell 42, 600-615.e604 (2017).

18. Bazellières, E. et al. Control of cell-cell forces and collective cell dynamics by the intercellular adhesome. Nat. Cell Biol. 17, 409-420 (2015).

19. Brugués, A. et al. Forces driving epithelial wound healing. Nat. Phys. 10, 683 (2014).

20. Kim, J.H. et al. Propulsion and navigation within the advancing monolayer sheet. Nat. Mater. 12, 856-863 (2013).

21. Cadart, C., Zlotek-Zlotkiewicz, E., Le Berre, M., Piel, M. \& Matthews, H.K. Exploring the function of cell shape and size during mitosis. Dev. Cell 29, 159-169 (2014).

22. Lancaster, O.M. et al. Mitotic rounding alters cell geometry to ensure efficient bipolar spindle formation. Dev. Cell 25, 270-283 (2013).

23. Cramer, L.P. \& Mitchison, T.J. Investigation of the mechanism of retraction of the cell margin and rearward flow of nodules during mitotic cell rounding. Mol. Biol. Cell 8, 109-119 (1997).

24. Maddox, A.S. \& Burridge, K. RhoA is required for cortical retraction and rigidity during mitotic cell rounding. J. Cell Biol. 160, 255-265 (2003).

25. Dao, V.T., Dupuy, A.G., Gavet, O., Caron, E. \& de Gunzburg, J. Dynamic changes in Rap1 activity are required for cell retraction and spreading during mitosis. $J$. Cell Sci. 122, 2996-3004 (2009).

26. Goody, M.F., Kelly, M.W., Lessard, K.N., Khalil, A. \& Henry, C.A. Nrk2bmediated NAD+ production regulates cell adhesion and is required for muscle morphogenesis in vivo: Nrk2b and NAD+ in muscle morphogenesis. Dev. Biol. 344, 809-826 (2010).

27. Sidhaye, J. \& Norden, C. Concerted action of neuroepithelial basal shrinkage and active epithelial migration ensures efficient optic cup morphogenesis. eLife $\mathbf{6}$, e22689 (2017).

28. Davoli, T. \& de Lange, T. The Causes and Consequences of Polyploidy in Normal Development and Cancer. Annu. Rev. Cell. Dev. Biol. 27, 585-610 (2011). 
29. Losick, Vicki P., Fox, Donald T. \& Spradling, Allan C. Polyploidization and Cell Fusion Contribute to Wound Healing in the Adult Drosophila Epithelium. Curr. Biol. 23, 2224-2232 (2013).

30. Campinho, P. et al. Tension-oriented cell divisions limit anisotropic tissue tension in epithelial spreading during zebrafish epiboly. Nat. Cell Biol. 15, 1405 (2013).

31. Lens, S.M.A. \& Medema, R.H. Cytokinesis defects and cancer. Nature Reviews Cancer 19, 32-45 (2019).

32. Fujiwara, T. et al. Cytokinesis failure generating tetraploids promotes tumorigenesis in p53-null cells. Nature 437, 1043 (2005).

33. Caldwell, C.M., Green, R.A. \& Kaplan, K.B. APC mutations lead to cytokinetic failures in vitro and tetraploid genotypes in Min mice. J. Cell Biol. 178, 1109-1120 (2007).

34. Riveline, D. et al. Focal Contacts as Mechanosensors. J. Cell Biol. 153, 1175-1186 (2001).

35. Elosegui-Artola, A. et al. Mechanical regulation of a molecular clutch defines force transmission and transduction in response to matrix rigidity. Nat. Cell Biol. 18, 540 (2016).

36. Oria, R. et al. Force loading explains spatial sensing of ligands by cells. Nature 552, 219 (2017).

37. Elosegui-Artola, A. et al. Rigidity sensing and adaptation through regulation of integrin types. Nat. Mater. 13, 631 (2014).

38. Chan, C.E. \& Odde, D.J. Traction dynamics of filopodia on compliant substrates. Science 322, 1687-1691 (2008).

39. Tambe, D.T. et al. Collective cell guidance by cooperative intercellular forces. Nat. Mater. 10, 469 (2011).

40. Uroz, M. et al. Regulation of cell cycle progression by cell-cell and cell-matrix forces. Nat. Cell Biol. 20, 646-654 (2018).

41. Dix, C.L. et al. The Role of Mitotic Cell-Substrate Adhesion Re-modeling in Animal Cell Division. Dev. Cell 45, 132-145. e133 (2018).

42. Kamranvar, S.A., Gupta, D.K., Huang, Y., Gupta, R.K. \& Johansson, S. Integrin signaling via FAK-Src controls cytokinetic abscission by decelerating PLK1 degradation and subsequent recruitment of CEP55 at the midbody. Oncotarget 7, 30820-30830 (2016).

43. Jones, M.C., Askari, J.A., Humphries, J.D. \& Humphries, M.J. Cell adhesion is regulated by CDK1 during the cell cycle. J. Cell Biol. 217, 3203-3218 (2018).

44. Lock, J.G. et al. Reticular adhesions are a distinct class of cell-matrix adhesions that mediate attachment during mitosis. Nat. Cell Biol. 20, 1290-1302 (2018).

45. Pellinen, T. et al. Integrin Trafficking Regulated by Rab21 Is Necessary for Cytokinesis. Dev. Cell 15, 371-385 (2008).

46. Taneja, N. et al. Focal adhesions control cleavage furrow shape and spindle tilt during mitosis. Sci. Rep. 6, 29846 (2016).

47. Bastos, R.N., Penate, X., Bates, M., Hammond, D. \& Barr, F.A. CYK4 inhibits Rac1-dependent PAK1 and ARHGEF7 effector pathways during cytokinesis. J. Cell Biol. 198, 865-880 (2012).

48. He, X., Chen, X., Li, B., Ji, J. \& Chen, S. FAK inhibitors induce cell multinucleation and dramatically increase pro-tumoral cytokine expression in RAW 264.7 macrophages. FEBS Lett. 591, 3861-3871 (2017). 
49. Burton, K. \& Taylor, D.L. Traction forces of cytokinesis measured with optically modified elastic substrata. Nature 385, 450-454 (1997).

50. Tanimoto, H. \& Sano, M. Dynamics of Traction Stress Field during Cell Division. Phys. Rev. Lett. 109, 248110 (2012).

51. Ermis, A. et al. Tetraploidization Is a Physiological Enhancer of Wound Healing. Eur. Surg. Res. 30, 385-392 (1998).

52. Schoenfelder, K.P. \& Fox, D.T. The expanding implications of polyploidy. J. Cell Biol. 209, 485-491 (2015).

53. Normand, G. \& King, R.W. Understanding Cytokinesis Failure. Adv. Exp. Med. Biol. 676, 27-55 (2010).

54. Zhang, W. \& Robinson, D.N. Balance of actively generated contractile and resistive forces controls cytokinesis dynamics. Proc. Natl. Acad. Sci. USA 102, 7186-7191 (2005).

55. Descovich, C.P. et al. Cross-linkers both drive and brake cytoskeletal remodeling and furrowing in cytokinesis. Mol. Biol. Cell 29, 622-631 (2018).

56. Polte, T.R., Eichler, G.S., Wang, N. \& Ingber, D.E. Extracellular matrix controls myosin light chain phosphorylation and cell contractility through modulation of cell shape and cytoskeletal prestress. Am. J. Physiol. Cell Physiol. 286, C518-C528 (2004).

\section{Acknowledgments}

We thank Natalia Castro and Cristina Garcia-Pastor for technical assistance, Caren Norden, Elisa Martí, and Ken Poss for sharing mutant zebrafish and Jose Muñoz's custom FEMplatform EMBRYO, which has been utilized to implement the numerical model. MU, AG$\mathrm{P}$, and IT were partially supported by pre-doctoral fellowships from the Spanish Ministry of Economy and Competitiveness (MINECO)/FEDER BES-2013-062633, BES-2013-064698, and FPU-AP2010-5071, respectively. This work was supported by the MINECO/FEDER (BFU2016-79916-P and BFU2014-52586-REDT to PR-C, BFU2015-65074-P to XT, SAF2015-69706-R to AR, BFU2016-75101-P and RYC-2014-15559 to VC), the Generalitat de Catalunya (2014-SGR-927 to XT, 2014-SGR-1460 and PERIS SLT002/16/00234 to AR, and the CERCA Programme), Instituto de Salud Carlos IIIISCIII/FEDER (Red de Terapia Celular - TerCel RD16/0011/0024 to AR), the European Research Council (CoG-616480 to XT), European Commission (Grant Agreeement SEP210342844 to PR-C and XT), Obra Social "La Caixa", a Career Integration Grant within the seventh European Community Framework Programme (PCIG10-GA-2011-303848 to PR-C), Fundació la Marató de TV3 (project 20153430 to AR and project 20133330 to PRC). IBEC is recipient of a Severo Ochoa Award of Excellence from the MINECO.

\section{Author contributions}

M.U., A.R. and X.T. conceived the study and designed experiments. M.U., A.G-P., I.T. JF.A. and A.M-L. performed experiments. M.U. and A.E-A analyzed data. A.E-A, V.C and P.R-C. carried out the theoretical modelling. M.U., S.P. and L.A. carried out STORM imaging. All authors discussed results. M.U. and X.T. wrote the manuscript with feedback from all authors. X.T. oversaw the project. 


\section{Ethics}

All experiments were performed in accordance with relevant guidelines and regulations, and conducted following procedures approved by the Ethics Committee on Experimental Animals of the PRBB (CEEA-PRBB).

\section{Competing interests}

The authors declare no competing interests.

\section{Data availability}

The data that support the findings of this study are available from the corresponding authors on reasonable request.

\section{Code availability}

Code use in this article can be made available upon request to the corresponding author. 


\section{Figures}

\section{Figure 1: An unconventional mechanism of collective migration.}

(a) Left: Illustration of the experimental model. Right: phase contrast image of the epicardial monolayer explanting from the heart. Scale bar, $150 \mu \mathrm{m}$. (b) Immunostaining of $\beta$-catenin (cyan) and Tcf-21 (gray) in epicardial explants. Left: cells at the leading edge. Right: cells behind the leading edge. Scale bar, $50 \mu \mathrm{m}$. (c) Left: Immunostaining of tubulin (gray) and nuclei (hoechst, cyan) in an expanding monolayer (heart at the top and leading edge at the bottom). Right: color-coded cell area. Scale bar, $50 \mu \mathrm{m}$. (d) Mean cell area as a function of the distance from the edge ( $n=1291$ cells from 4 hearts). Error bars represent SD. (e) Mean nuclear area as a function of the distance from the edge $(n=1291$ cells from 4 hearts). Error bars represent SD. f-g, Immunostaining of $\beta$-catenin (red), phospho-paxillin (gray) and F-actin (green) in epicardial cells. (f) Merge images. Left: follower cells. Center and right: leader cells. Scale bar, $20 \mu \mathrm{m}$. (g) Magnified view of the blue squares highlighted

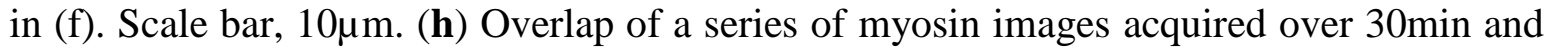
color coded according to acquisition time. Note that basal stress fibers appear white, indicating that they are slow, whereas the cell-cell network and lamellipodia are fast. Left: follower cells. Right: leader cells. Scale bar, $20 \mu \mathrm{m}$. Images are representative of two (b), four (c), eight $(\mathbf{f}, \mathbf{g})$, or six (h) hearts.

\section{Figure 2: Mechanics of epicardial migration}

(a) Traction vector map overlaid on a phase contrast image. Dashed lines mark the heart edge (black) and the leading edge of the monolayer (white). Scale bar, 50 $\mu$ m. (b) Mean parallel and perpendicular components (unsigned) of the traction vector with respect to the leading edge ( $n=5$ monolayers from 4 hearts). Inset: average parallel and perpendicular components (signed) of the traction vector with respect to the leading edge $(n=5$ monolayers from 4 hearts). Positive radial tractions point towards the heart. Shaded areas represent SD. (c) Traction map overlaid on an inverted myosin fluorescence image. Scale bar, $20 \mu \mathrm{m}$. (d) Scheme of the one-dimensional in silico model for a unidimensional epithelium of elastic epicardial cells sliding viscously on a rigid substrate in response to a self-propelling force at the leading-edge node. (e) Schematic of the nodal force balance in the model. Forces are represented through arrows and all act at the generic junction node $x_{i}$ (for the sake of the clarity, they have been drawn as acting at points in the proximity of node $x_{i}$ ). Self-propelling force $\mathbf{F}_{i}$ is in yellow; elastic forces $\mathbf{f}_{i}{ }^{e}$ and $\mathbf{f}_{i+1}{ }^{e}$ are in green; and, viscous force $\mathbf{f}_{i}^{\mathrm{V}}$ is in gray. $\mathbf{f}-\mathbf{g}$, In vitro and in silico normalized cellular lengths at 0 (f) and 15 (g) hours as a function of the distance from the epithelial migrating edge. Lengths are normalized to the average initial length. Error bars represent SD. Experimental data in $\mathbf{f}, \mathbf{g}$ corresponds to one monolayer expansion $(\mathrm{n}=41$ cells at $\mathrm{t}=0 \mathrm{~h}$ and $\mathrm{n}=88$ cells at $\mathrm{t}=15 \mathrm{~h}$ ) representative of $\mathrm{n}=3$ hearts. Images are representative of four (a) or six (c) hearts.

\section{Figure 3: Epicardial cells exert traction forces during cytokinesis}


(a) Fluorescence staining of $\beta$-catenin (gray) and nuclei (hoechst, cyan) in cells at the onset of metaphase and anaphase. Scale bar, 10um. (b) Left: Fluorescence staining of myosin (green), tubulin (magenta) and nuclei (hoechst, blue) of a cell in metaphase. Scale bar, $10 \mu \mathrm{m}$. Right: YZ projection of the merge image along the orange dashed line illustrates that cells remain flat during division. Scale bar, $2 \mu \mathrm{m}$. (c) Mean cell area (left axis) and eccentricity (right axis) of dividing cells up to anaphase (labelled as $t=0 \mathrm{~min}$ ). Shaded areas represent SD ( $\mathrm{n}=59$ cells from 4 hearts). (d) Time evolution of tractions of a dividing

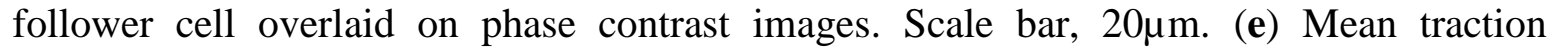
modulus during cell division. Tractions were averaged in a region of varying size enclosing the cytokinetic ring (illustrated in Supplementary Video 10). Dashed line represents the beginning of anaphase. Shaded areas represent SEM ( $n=59$ cells from 4 hearts). (f) Tractions during division overlaid on myosin fluorescence images. Gray dashed line labels the images in which the cytokinetic ring is clearly present. Scale bar, $10 \mu \mathrm{m}$. (g) Fluorescence staining of tubulin (magenta), myosin (green), phospho-paxillin (yellow) and nuclei (hoechst, blue) of a cell during cytokinesis. White dashed line marks the outline of

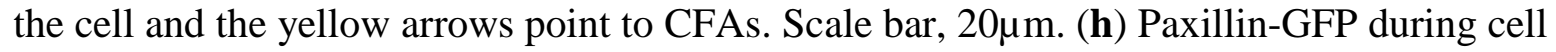
division. The green dashed lines outline the nuclei. Scale bar, $10 \mu \mathrm{m}$. The yellow dashed lines mark the area rotated and zoomed at the bottom of each image. Yellow arrows point at CFAs. Scale bar, $2.5 \mu \mathrm{m}$. (i) Left: Fluorescence staining of tubulin (green), F-actin (red), paxillin (yellow) and nuclei (hoechst, blue) of a cell during cytokinesis. Scale bar, $10 \mu \mathrm{m}$. Right: STORM image of F-actin at the interface between the two daughter cells (corresponding to the region marked with blue dash lines on the left image). Epifluorescence of paxillin is overlaid on the STORM image. Scale bar, 10 $\mu \mathrm{m}$. In all graphs, the origin of the time axis $(0 \mathrm{~min})$ is defined as the last time point in which only one nucleus was visible. Images shown in a are representative of $n=9$ divisions from three hearts, images shown in $\mathbf{b}$ are representative of $n=9$ divisions from four hearts, images shown in $\mathbf{d}$ are representative of $n=59$ divisions from four hearts, images shown in $\mathbf{f}$ are representative of $n=29$ divisions from four hearts, images shown in $\mathbf{g}$ are representative of $n=26$ divisions from nine hearts inspected in higher resolution in $\mathbf{i}$, and images shown in $\mathbf{h}$ are representative of $n=13$ divisions from two hearts.

\section{Figure 4: A large number of cells are multinucleated due to cytokinetic failure}

(a) Fluorescence staining of $\beta$-catenin (cyan) and nuclei (hoechst, gray) in an epicardial explant. Scale bar, $50 \mu \mathrm{m}$. (b) Percentage of binucleated cells as a function of the distance from the edge $(n=1291$ cells from 4 hearts). (c) Fluorescence staining of $\beta$-catenin (green) and nuclei (hoechst, magenta) at the surface of a regenerating heart (3 days post amputation). Scale bar, $20 \mu \mathrm{m}$. (d) Detail of a cell in (c) with three nuclei. Left: merge. Center: $\beta$-catenin. Right: nuclei. Scale bar, $10 \mu \mathrm{m}$. Bottom: Projections along the lines labelled 1 (YZ, yellow) and 2 (blue, XZ) in the merge image. Horizontal scale bar, $2 \mu \mathrm{m}$. Vertical scale bar, $2 \mu \mathrm{m}$. (e) Time evolution of tractions during a failed cell division overlaid on myosin fluorescence images. The origin of the time axis (0min) is defined as the last time point in which only one nucleus was visible. Green lines outline the nuclei.

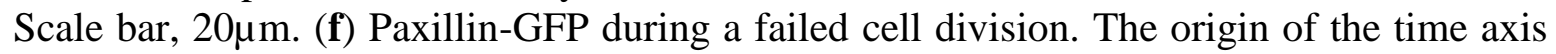
(0min) is defined as the last time point in which only one nucleus was visible. Green lines outline the nuclei. Scale bar, $20 \mu \mathrm{m}$. The yellow dashed lines mark the area rotated and zoomed at the bottom of each image. Scale bar, $5 \mu \mathrm{m}$. Images are representative of four (a) 
or two $(\mathbf{c}, \mathbf{d})$ hearts. Images shown in e are representative of $\mathrm{n}=9$ failed divisions from four hearts and images shown in $\mathbf{f}$ are representative of $n=5$ failed divisions from two hearts.

\section{Figure 5: Reinforcement of CFAs leads to cytokinetic failure}

$\mathbf{a}-\mathbf{b}$, Time evolution of the normalized mean myosin intensity during division for successful and failed divisions on $14 \mathrm{kPa}$ substrates (a), and on $5 \mathrm{kPa}$ substrates (b). c-e Scheme of the clutch model. The basal segment of the cytokinetic ring is modeled as a 1D contractile cable containing actin (red) and myosin (gray) anchored to the substrate at the edges by integrins (light brown) and adaptor proteins (teal). (c) Model for successful divisions. (d) Model for failed divisions. (e) The molecular-clutch model. f-g, Predicted (f) and experimental (g) time evolution of the mean traction force on $14 \mathrm{kPa}$ substrates for successful and failed divisions. (h) Experimental traction force $v s$ ring contraction velocity for the first time points of closure (up to 6 min after anaphase for successful divisions and 9 min for failed divisions) on $14 \mathrm{kPa}$ substrates. $\mathbf{i}-\mathbf{j}$, Predicted (i) and experimental (j) time evolution of the mean traction force on $5 \mathrm{kPa}$ substrates for successful and failed divisions. (k) Experimental traction force $v s$ ring contraction velocity for the first time points of closure (up to $6 \mathrm{~min}$ after anaphase for successful divisions and $9 \mathrm{~min}$ for failed divisions) on $5 \mathrm{kPa}$ substrates. (l) Mean loading rate for successful and failed divisions on $14 \mathrm{kPa}$ and $5 \mathrm{kPa}$ substrates. Error bars represent SD. $* P=0.0166$ and $* * * P=0.0002$, two-tailed MannWhitney test. Myosin and tractions were averaged in a region enclosing the cytokinetic ring (illustrated in Supplementary Video 10). In a,b,g,j shaded areas represent SEM and dashed line represents the beginning of anaphase. Dashed lines in $\mathrm{h}, \mathrm{k}$ represent linear fits. For $14 \mathrm{kPa} n=29$ successful and 9 failed divisions from 4 hearts, and for $5 \mathrm{kPa} n=39$ successful and 13 failed divisions from 5 hearts. (m) Fibronectin (imaged through fluorescent

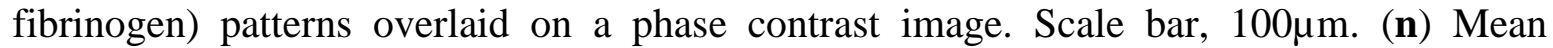
percentage of division failure in the areas of high and low ECM coating $(n=10$ hearts from 6 independent experiments). $* P=0.019$, paired two tailed t-test. Error bars represent SD. (o) Left: merge image of the fluorescent fibrinogen (green), an immunostaining of phospho-paxillin (gray) and nuclei (hoechst, blue) in epicardial cells. Right: immunostaining of phospho-paxillin. Scale bar, $50 \mu \mathrm{m}$. Images are representative of ten $(\mathbf{m})$ or eight $(\mathbf{o})$ hearts. 


\section{Methods}

\section{Zebrafish husbandry and surgical procedures}

All experiments were carried out with adult zebrafish between 3 and 15 months of age, maintained and raised according to standard protocols ${ }^{1}$. In this study, the following zebrafish strains were used: wild-type $\mathrm{AB}, \operatorname{Tg}(a c t b 2: m y l 12.1$-EGFP) (obtained from Elisa Martí's lab) and Tg(bActin:Paxilin-GFP) ${ }^{+/-}$(obtained from Caren Norden's Lab).

For the 3dpa hearts, the ventricular amputation was performed as previously described by Raya et. al. ${ }^{2}$. All animal procedures were approved by the Ethics Committee on Experimental Animals of the PRBB (CEEA-PRBB).

\section{Preparation of polyacrylamide gel substrates}

Glass-bottom dishes were activated by using a 1:1:14 solution of acetic acid/bind-silane (M6514, Sigma)/ethanol. The dishes were washed twice with ethanol and air-dried for 5 min. For $14 \mathrm{kPa}(5 \mathrm{kpa})$ gel substrates, a $500 \mu 1$ stock solution containing Hepes $10 \mathrm{mM}$, $93.75 \mu \mathrm{l}(68.75 \mu \mathrm{l})$ acrylamide $40 \%$ (161-0140, BioRad), 35 $\mu \mathrm{l}(22.5 \mu \mathrm{l})$ bisacrylamide $2 \%$

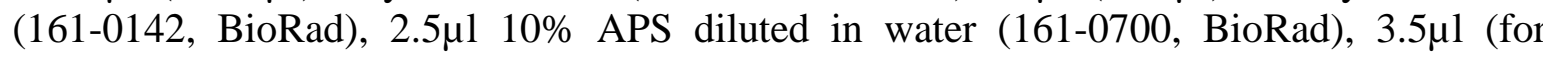
imaging with $20 \times$ objectives -or $12 \mu \mathrm{l}$ for $60 \times$ objectives-) 200 -nm-diameter far red fluorescent carboxylate-modified beads (F8807, ThermoFisher) and 0.25 $\mu$ 1 TEMED (T9281, Sigma-Aldrich, added last) was prepared. A drop of $18 \mu \mathrm{l}$ was added to the center of the glass-bottom dishes and the solution was covered with 18-mm-diameter GelBond film (Lonza) coverslips (hydrophobic side down) custom cut by an electronic cutting tool (Silhouette Cameo). After polymerization, the coverslip was removed, and gels were functionalized using sulfo-sanpah. For this purpose, a drop of $80 \mu \mathrm{l}$ of sulfo-sanpah was placed on top of the polyacrylamide gel and activated by UV light for $3 \mathrm{~min}$. Sulfo-sanpah (22589, Thermo-Scientific) was diluted in miliQ water to a final concentration of $2 \mathrm{mg} / \mathrm{ml}$ from an initial dilution of $50 \mathrm{mg} / \mathrm{ml}$ in DMSO kept at $-80^{\circ}$. Next, gels were washed twice with miliQ water and once with PBS for $5 \mathrm{~min}$ each. Afterwards, gels were incubated with $200 \mu \mathrm{l}$ of either a $0.1 \mathrm{mg} / \mathrm{ml}$ collagen I solution $(60-30-810$, Firstlink) or a $10 \mu \mathrm{g} / \mathrm{ml}$ fibronectin solution (F0895, Sigma-Aldrich) overnight at $4^{\circ} \mathrm{C}$.

\section{Epicardium explant culture}

Before seeding, the gels coated with ECM were washed twice with PBS, sterilized with UV (30min), covered with media, and kept in the incubator at $28^{\circ}$. Simultaneously, $18 \mathrm{~mm} \varnothing$ glass coverslips were sonicated for $5 \mathrm{~min}$ in acetone and $5 \mathrm{~min}$ in isopropanol, $\mathrm{N}_{2}$ dried and sterilized with UV for 20min.

After an intra-abdominal injection of $20 \mu \mathrm{l}$ of Heparin (1000U/ml, Sigma), zebrafish were sacrificed with $0,6 \%$ tricaine in fish water. Hearts were collected and dissected in a $10 \%$ Penicilin/Streptomycin (ThermoFisher, 10378-016), 10\% Fungizone (ThermoFisher, 15290-026) PBS solution (at $28^{\circ}$ ). The media was removed from the gels and the hearts were seeded on top with a small drop of media. A clean sterile coverslip was placed on top 
of the heart and, after 1 minute, $2 \mathrm{ml}$ of media were added. Finally, the wells were incubated at $28^{\circ}$.

The epicardial cell explants were kept in L-15 cell culture medium supplemented with 4\% FBS (ThermoFisher, 10270-106), 2\% Pen/Strep, and 2\% Fungizone. The media was changed daily. After 3-5 days, epicardial cells started to explant in $\sim 50 \%$ of the hearts.

\section{Fluorescence staining}

Epicardial cells on $14 \mathrm{kPa}$ substrates were fixed with $4 \%$ paraformaldehyde for 15 min (heating the PFA to $28^{\circ} \mathrm{C}$ and without washing previously) and permeabilized in $0.5 \%$ Triton X-100 for 7 min. Cells were blocked in 10\% FBS for $1 \mathrm{~h}$ before being incubated for $3 \mathrm{~h}$ with primary antibodies. After incubation with the appropriate fluorescence-conjugated secondary antibodies for $1 \mathrm{~h}$, cells were washed several times and mounted in Mowiol reagent. A 1:5000 Hoechst (ThermoFisher, H3570) solution in PBS was added after removing the secondary antibody for 10 minutes. If required, also a 1:1000 phalloidinTRITC (Sigma-Aldrich, P1951) solution in PBS was added for 30 minutes. For staining the full heart 3dpa, the same protocol was followed maintaining the heart immersed in the different solutions.

\section{$\underline{\text { Antibodies }}$}

The primary antibodies used were: Tcf-21 rabbit antibody (Abcam, ab49475; concentration 1:200), $\beta$-catenin mouse antibody (BD Biosciences, 610154; concentration 1:400), phospho-paxillin rabbit antibody (Cell Signalling, 2541S; 1:100 concentration), $\alpha$ -tubulin mouse antibody (Sigma-Aldrich, T5168; 1:400 concentration), tubulin rat antibody (Abcam, ab6160; 1:400/1:600 concentration) and diphosphorylated-Myosin Light Chain rabbit antibody (Cell Signalling, 3674S; 1:50 concentration).

The secondary antibodies used were: Alexa Fluor 488 anti-rabbit (ThermoFisher, A21206), Alexa Fluor 555 anti-rabbit (ThermoFisher, A21429), Alexa Fluor 647 anti-rabbit (ThermoFisher, A21245), Alexa Fluor 488 anti-mouse (ThermoFisher, A11029), Alexa Fluor 555 anti-mouse (ThermoFisher, A21424) and Alexa Fluor 647 anti-rat (ThermoFisher, A21247). All secondary antibodies were diluted 1:200.

\section{Micropatterning}

Glass-bottom petri dishes (P35-0-20, MatTek) were micropatterned using the PRIMO system (Alveole) mounted on an inverted microscope (Nikon Eclipse Ti). The petri dish glass surfaces were cleaned with $96 \%$ ethanol and plasma cleaned for 30s. Immediately after plasma cleaning a PDMS stencil of approximately $1 \times 1 \times 0.5 \mathrm{~cm}$ with a cylindrical opening of $6 \mathrm{~mm} \varnothing$ was bound to the glass. A $0.1 \mathrm{mg} / \mathrm{ml}$ solution of Pll-g-Peg (PLL(20)$\mathrm{g}[3.5]-\mathrm{PEG}(2 \mathrm{kDa}), \mathrm{SuSoS})$ in PBS was added in the cylindrical opening and incubated at room temperature for $1 \mathrm{~h}$ (or up to $24 \mathrm{~h}$ in the fridge). The Pll-g-Peg solution was washed 3 times with filtered PBS and $10 \mu$ of the PRIMO photoinitiator (PLPP, Alveole) were added and covered with a $18 \mathrm{~mm} \varnothing$ glass coverslip (placed on top of the PDMS to avoid 
evaporation of the photo initiator). Then, the circular 6mmø area coated with PLL-g-PEG and containing the photoinitiator was patterned with stripes of $90 \mu \mathrm{m}$ by using the PRIMO system with the Leonardo software (Alveole). For this procedure, a 20× CFI S Plan Fluor ELWD ADM objective and a power of $900 \mathrm{~mJ} / \mathrm{mm}^{2}$ were used. Once the patterning was completed, the photoinitiator was cleaned 3 times with filtered PBS and $20 \mu 1$ of a solution of 8\% fibronectin (F0895, Sigma-Aldrich) and 2\% Alexa Fluor 647 fibrinogen (F35200, ThermoFisher) was incubated on the patterned substrate for 5min. After washing 3 times with filtered PBS, the PDMS stencil was removed and $2 \mathrm{ml}$ of PBS were added. Patterned petri dishes were stored in the fridge up to $72 \mathrm{~h}$ before zebrafish heart seeding. A few hours before seeding, PBS was replaced by cell media and they were kept at $28^{\circ}$.

\section{Stochastic Optical Reconstruction Microscopy (STORM) Imaging}

For STORM imaging, hearts were seeded on glass petri dishes, and F-actin was labeled with phalloidin-647 (ThermoFisher, A22287) at 1:1000 dilution in PBS. Additional immunostaining was performed using the following primary antibodies: Paxillin mouse antibody (BD Biosciences, 610051) and tubulin rat antibody (Abcam, ab6160). Additional secondary antibodies were: Alexa Fluor 488 anti-rat (ThermoFisher, A11006) and Alexa Fluor 555 anti-mouse (ThermoFisher, A21424). All antibodies were diluted 1:1000.

Images were acquired using a Nikon N-STORM 4.0 system configured for total internal reflection fluorescence (TIRF) imaging. Excitation inclination was tuned to adjust focus and to maximize the signal-to-noise ratio. For STORM imaging Alexa647 was excited illuminating the sample with the $647 \mathrm{~nm}(\sim 160 \mathrm{~mW})$ laser line built into the microscope. Fluorescence was collected by means of a Nikon $\times 100,1.4$ NA oil immersion objective and passed through a quad-band-pass dichroic filter (97335 Nikon). Images were recorded on a $256 \times 256$ pixel region (pixel size $160 \mathrm{~nm}$ ) of a sCMOS camera (Hamamatsu). Samples were kept in Gloxy buffer for STORM imaging as previously described ${ }^{57}$. Single-molecule localization sequences were analyzed with the STORM plug-in of NIS element Nikon software.

\section{Measurement of cell height}

For cell height measurements hearts were fixed with a solution of $4 \%$ paraformaldehyde and $0.4 \%$ glutaraldehyde for $10 \mathrm{~min}$ (heating the solution to $28^{\circ} \mathrm{C}$ and without washing previously). Cell membranes were stained with WGA-AF647 (ThermoFisher, W32466) at $1 \mu \mathrm{g} / \mathrm{mL}$ for $10 \mathrm{~min}$. Then samples were imaged in the same conditions as for actin imaging but acquiring 3D stacks. Stacks were collected for each image covering over the top of the cell with $80 \mathrm{~nm}$ steps and 10000 frames/step at 10ms integration time.

3D STORM data localization and fitting was performed using the STORM module of NISElements using a Gaussian fitting. The first 50 frames were discarded, due to incomplete photo-switching. This analysis yielded a molecule list in binary format from which multiple emitters are automatically discarded prior to analysis. To avoid overcounting, blinkings detected in consecutive frames are counted as single by the software. 3D data 
reconstruction was represented in Matlab using a custom-made script for calculating maximum height, height from the leading edge and the height profile between cells.

\section{$\underline{\text { Time lapse imaging }}$}

Multidimensional acquisition routines were performed on an automated inverted microscope (Nikon Eclipse Ti) equipped with thermal control, $\mathrm{CO}_{2}$ and humidity control, using MetaMorph (Universal Imaging) software. All WT hearts were imaged using a 20x objective with a $5 \mathrm{~min}$ time interval.

\section{Spinning-Disk imaging}

An inverted Nikon microscope with a spinning disk confocal unit (CSU-WD, Yokogawa) and Zyla sCMOS camera (Andor) was used for high-resolution image acquisition. All transgenic hearts were imaged using a 60x objective with a 3 min time interval.

\section{$\underline{\text { Traction microscopy }}$}

Traction forces were computed using Fourier transform-based traction microscopy with a finite gel thickness. Gel displacements between any experimental time point and a reference image obtained after monolayer trypsinization were computed using home-made particle imaging velocimetry software ${ }^{13}$.

\section{Cell/nuclear area measurements}

For the measurements in Fig. 1d-e, cells and nuclei were segmented using a custom-made semi-automatic watershed algorithm applied to tubulin and nuclear fluorescence stainings. From this analysis, both the cellular/nuclear areas and the number of nuclei per cell as a function of the distance from the edge were computed. For the measurements in Fig. $2 \mathrm{f}-\mathrm{g}$, cell shapes were manually drawn from the phase contrast images, and normalized cell lengths were computed as:

$l=\sqrt{A / A_{t=0 h, d=125 \mu m}}$

where $l$ is the normalized cell length, $A$ is the cell area and $A_{t=0 h, d=125 \mu m}$ is the mean cell area at time $0 \mathrm{~h}$ and at $125 \mu \mathrm{m}$ from the edge.

\section{$\underline{\text { Loading rate and ring velocity }}$}

Loading rate $\dot{T}$ was defined for each cell as:

$\dot{T}=\frac{\overline{|T|}_{t_{2}}-\overline{|T|}_{t_{1}}}{t_{2}-t_{1}}$ 
where $\overline{|T|}_{t_{1}}$ and $\overline{|T|}_{t_{2}}$ are the traction moduli evaluated at times $t_{1}$ and $t_{2}\left(t_{2}=12 \mathrm{~min}\right.$ and $\left.t_{1}=3 \mathrm{~min}\right)$. The origin of time $\mathrm{t}=0 \mathrm{~min}$ is defined as the last time point in which only one nucleus was visible.

Ring velocity at time point $i\left(v_{i}\right)$ was calculated as:

$v_{i}=\frac{l_{i+1}-l_{i}}{\Delta t}$

where $l_{i}$ and $l_{i+1}$ are the length of the ring at the time points $i$ and $i+1$, respectively, and $\Delta t$ is the time interval of acquisition between images $i$ and $i+1$ ( $3 \mathrm{~min}$ ). Velocity was calculated during the time span in which the length of the ring was accurately measurable (up to 6min after anaphase for successful divisions and up to $9 \mathrm{~min}$ after anaphase for failed divisions).

\section{Formulation and solution of the cell migration model}

Our 1D in silico model reiterates a previous formulation of ours ${ }^{10}$ by representing the epicardium as a series of elastic cells, which have constant stiffness $k$ and are joint at nodal intercellular junctions (Fig. 2d). Each cell can generate a self-propelling force $\boldsymbol{F}_{i}$ that pushes the cell forward in the direction of motion. This force results from the average cellular traction $\boldsymbol{T}_{i}$ (force per unit area of substrate interface), which each cell can exert on the substrate in the direction opposite to the direction of motion. The propelling force $\boldsymbol{F}_{i}$ can be transmitted to neighbouring cells through intercellular junctions throughout the epicardium. Transmission of propulsion at each intercellular junction is mediated by elastic and viscous forces (Fig. 2d). Elastic forces are generated by compliant cells, which are represented as elastic springs connecting intercellular junctions. Different types of friction have been considered in monolayers ${ }^{10,58}$. For simplicity, here we assumed that frictional forces are generated by the underlying viscous substrate, which is represented as a dashpot of constant viscosity $\eta$ connected to a fixed node, which result in a viscous drag opposing cellular motion at intercellular junctions. In the quasi-static approximation, the force balance at a generic junctional node $x_{\mathrm{i}}$ (Fig. 2e) sums to nought these forces: the selfpropulsion $\mathbf{F}_{i}$, the elastic responses $\mathbf{f}_{i}^{e}=k \varepsilon_{i} \widehat{\boldsymbol{\omega}}$ and $\mathbf{f}_{i+1}^{e}=-k \varepsilon_{i+1} \hat{\boldsymbol{\omega}}$ of the two cells joined at node $x_{\mathrm{i}}$ and the viscous friction $\mathbf{f}_{i}^{v}=\eta \dot{\varepsilon}_{i} \widehat{\boldsymbol{\omega}}$ between those two cells and the substrate interface (Fig. 2e). Here $\varepsilon_{i}$ denotes the strain undergone by the cellular spring connecting nodes $x_{i-1}$ and $x_{i}$ and $\dot{\varepsilon}_{i}$ denotes the strain rate of the dashpot connecting node $x_{i}$ to the substrate, whereas $\widehat{\boldsymbol{\omega}}$ is the unit vector pointing in the one-dimensional direction of motion. Thus, force balance at each node $x_{i}$ reads:

$F_{i}+k \varepsilon_{i}-k \varepsilon_{i+1}-\eta \dot{\varepsilon}_{i}=0$.

A numerical solution of the model is obtained though Newton-Raphson reiterations. The magnitudes of the propelling force $F$, elastic stiffness $k$ and viscous drag $\eta$ utilised to simulate the epicardial monolayer were chosen based on experimental data. Specifically, the in silico epicardial monolayer was assumed to approximately include 9 cellular units 
having initial length $30 \mu \mathrm{m}$. From average traction and cellular area at the leading edge we estimated a self-propelling force of $250 \mathrm{nN}$. From this force value and experimental measurements of $\varepsilon$ and $\dot{\varepsilon}$ we estimated $\eta_{\mathrm{c}}=1.3 \times 10^{5} \mathrm{nN}$.min and $k_{\mathrm{c}}=312 \mathrm{nN}$. In a previous implementation of the model ${ }^{10}$, non-linearities were added to capture the propagation of mechanical waves. No clear sign of wave propagation was observed here and those nonlinearities were not included in the present model implementation.

\section{Motor clutch model}

The computational model was implemented by Montecarlo simulation as previously explained in detail ${ }^{35-37}$. Specifically, the implementation used is the same one as that described in ${ }^{37}$, with the only difference that only one population of integrins is considered rather than two. We thus refer the reader to the supplementary information of that publication for details. The model considers a given number of myosin motors $n_{m}$, each able to exert a force $F_{m}$, pulling on an actin filament. The actin filament is bound to the ECM molecules $n_{c l}$ on the substrate through molecular clutches composed of integrins and adaptor proteins. The ECM molecules are connected in parallel to the substrate, which is represented as a spring with a spring constant $k_{s u b}$, representing substrate rigidity. In the absence of adhesion, when there is no load as no clutch is engaged, the actin filament moves freely with a speed $v_{a}$. At each time step, each ECM molecule can engage any unbound clutch according to a binding rate $k_{o n}=k_{\text {ont }} * d_{c l u}$, where $k_{\text {ont }}$ is the binding rate that characterizes the integrin-ECM interaction and $d_{c l u}$ is the density of clutches. Likewise, each clutch bound to ECM can unbind according to an unbinding rate $k_{\text {off }}$ dependent on force as a catch bond. To implement reinforcement of adhesions, the computational model increases the number of clutches by an amount $d_{a d d}$ (which leads fundamentally to an increase in the binding rate $k_{o n}$ ) if a clutch is submitted to a load higher than the reinforcement force $F_{r}$. Similarly, the number of integrins will decrease by the same amount if a clutch is submitted to a load lower than the reinforcement threshold. This implementation takes into account the observation that adhesions grow when submitted to force and shrink when force is low ${ }^{34,59}$. However, since the initial basal clutch density represents the number of clutches before any significant adhesion growth, the density of clutches is not allowed to decrease below this value. This explicit consideration of reinforcement, which distinguishes our approach from other thorough theoretical descriptions of adhesion ${ }^{60-63}$, is fundamental to model our results.

At each time step, the actin filament contracts with a speed $v_{a}$, displacing all the bound clutches by the same amount, increasing the force and changing $k_{\text {off }}$. The total force on the substrate $F_{\text {sub }}$ is recalculated at every time step imposing force balance as:

$F_{\text {sub }}=\frac{\kappa_{\text {sub }} k_{c} \sum_{i=1}^{n_{\text {bound }}} x_{i}}{\kappa_{\text {sub }}+n_{\text {bound }} \kappa_{c}}$

Where $x_{i}$ is the position of each clutch, $k_{c}$ is the spring constant of each clutch, and $n_{\text {bound }}$ is the number of bound clutches at that time step. Then, actin speed $v_{a}$ is recalculated by assuming a linear dependence on force as 
$v_{a}=v_{u}\left(1-\frac{F_{s u b}}{n_{m} F_{m}}\right)$

$v_{a}$ ranges from $v_{u}$ (in the absence of force) to 0 (when force is $n_{m} * F_{m}$ ). The simulation is run for each experimental point for time steps of $5 \mathrm{~ms}$ for 15000 s to assure that steady state is reached. After the simulation finishes, $F$ and $K_{s u b}$ are converted into cell traction stresses $P_{s u b}$ and Young's moduli $E$, respectively, by assuming a given adhesion radius $r_{a}$ as described previously ${ }^{35,37,64}$. Specifically:

$E=\frac{9 k_{\text {sub }}}{4 \pi r_{a}}$
$P_{\text {sub }}=\frac{F_{\text {sub }}}{\pi^{2} r_{a}}$

For the computations shown in Figure 5 all the parameters are kept constant for both stiffnesses, even the initial number of myosin motors $n_{m 0}$, but the number of myosin motors varies with time based on the experimental data. To ensure that this model input varies smoothly with time, experimental data are fitted with a sum of Gaussian functions. Model outputs in Fig. 5 and Fig. S3 are steady state predictions of time-averaged force and actin speed values. We therefore note that the time axis in model predictions in Figs. 5 and S3 merely represents steady-state averages corresponding to the myosin motors associated to that time point in experimental measurements. For the computations shown in supplementary Fig. 3, all the parameters are kept constant except the reinforcement threshold $F_{r}$. Model parameters can be found in Supplementary Table 1.

\section{Methods references}

57. van der Zwaag, D. et al. Super Resolution Imaging of Nanoparticles Cellular Uptake and Trafficking. ACS Appl. Mater. Interfaces 8, 6391-6399 (2016).

58. Christensen, A. et al. Friction-limited cell motility in confluent monolayer tissue. Phys. Biol. 15, 066004 (2018).

59. Balaban, N.Q. et al. Force and focal adhesion assembly: a close relationship studied using elastic micropatterned substrates. Nat. Cell Biol. 3, 466 (2001).

60. Srinivasan, M. \& Walcott, S. Binding site models of friction due to the formation and rupture of bonds: state-function formalism, force-velocity relations, response to slip velocity transients, and slip stability. Phys. Rev. E 80, 046124 (2009).

61. Sabass, B. \& Schwarz, U.S. Modeling cytoskeletal flow over adhesion sites: competition between stochastic bond dynamics and intracellular relaxation. $J$. Phys.: Condens. Matter 22, 194112 (2010).

62. Harland, B., Walcott, S. \& Sun, S.X. Adhesion dynamics and durotaxis in migrating cells. Phys. Biol. 8, 015011 (2011).

63. Sens, P. Rigidity sensing by stochastic sliding friction. Europhys. Lett. 104, 38003 (2013).

64. Ghibaudo, M. et al. Traction forces and rigidity sensing regulate cell functions. Soft Matter 4, 1836-1843 (2008). 
a

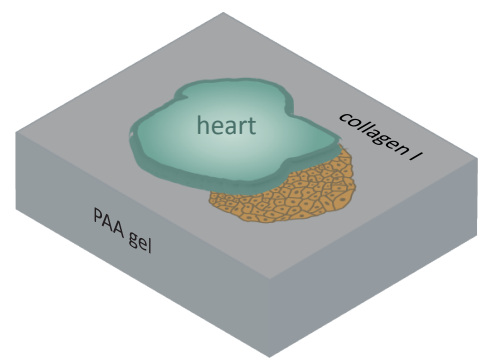

b
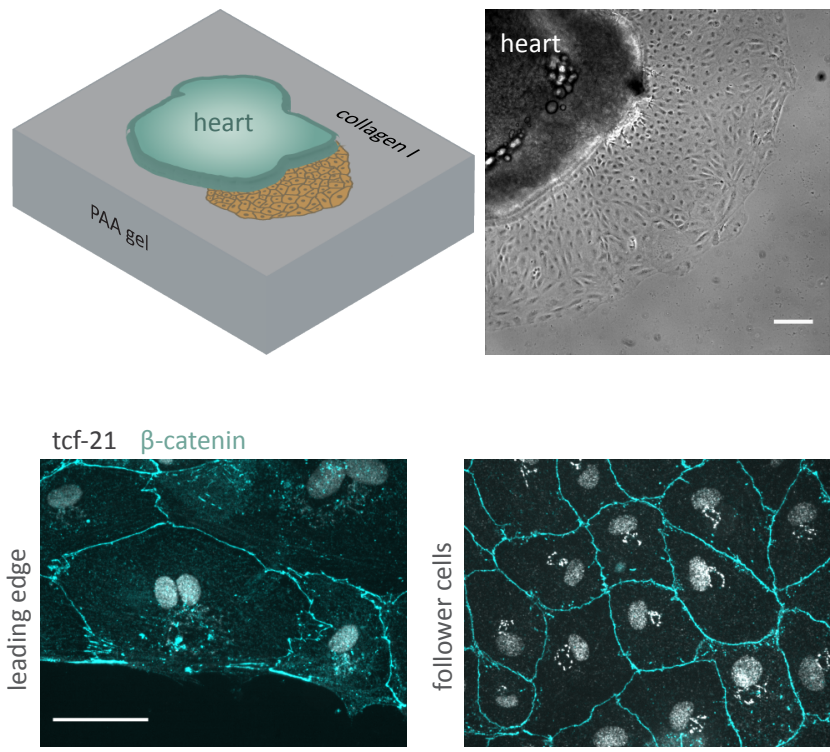

C

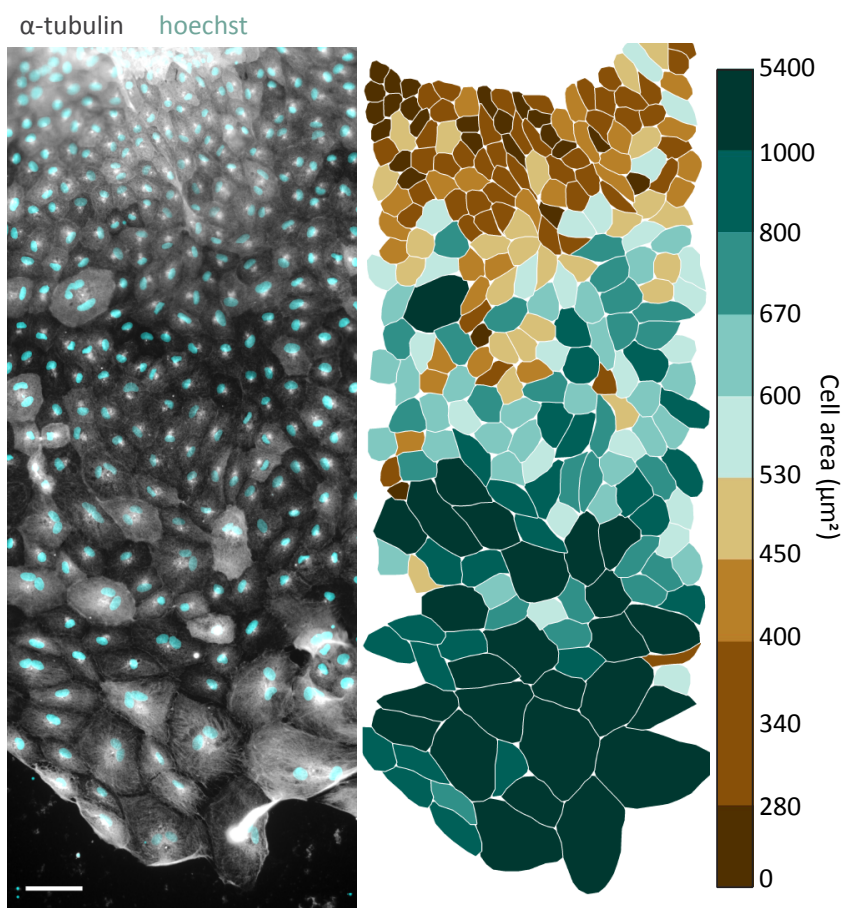

d
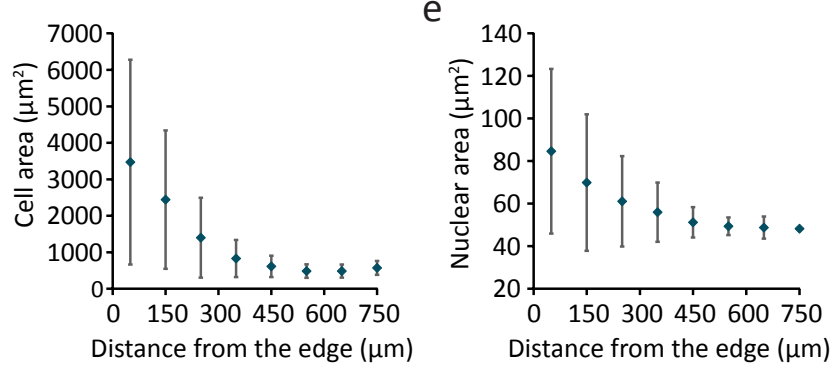

f

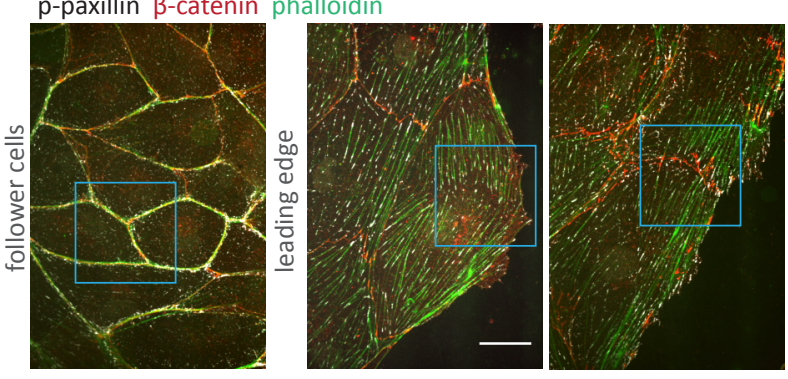

g

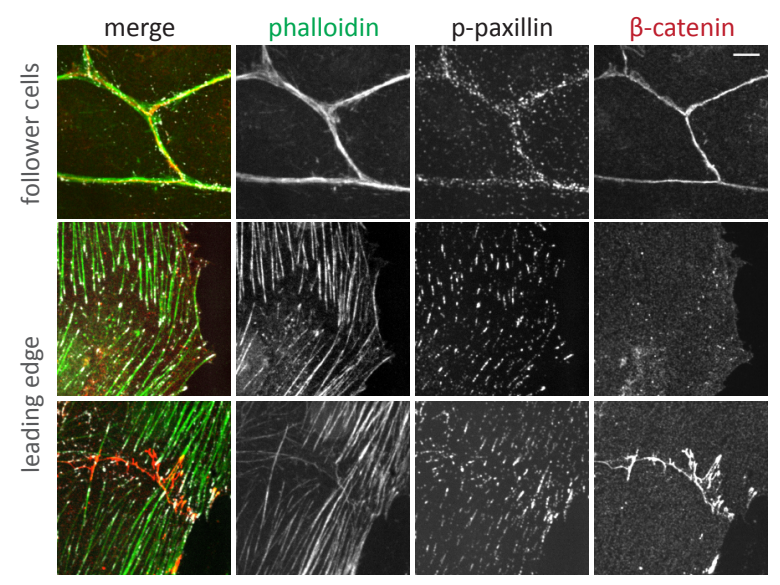

h

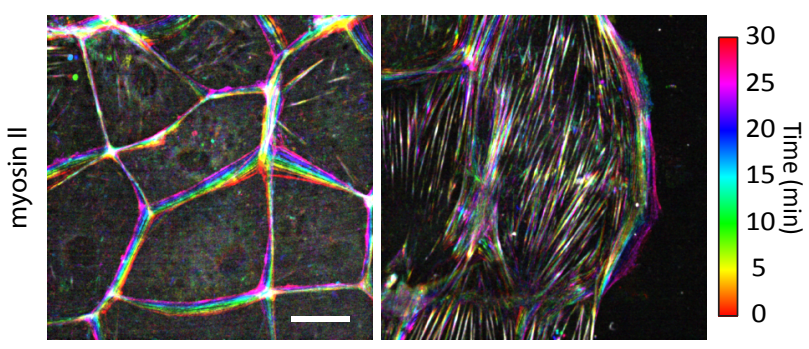


a

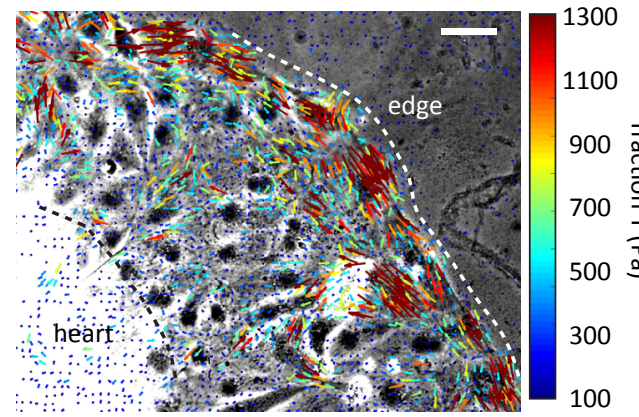

d

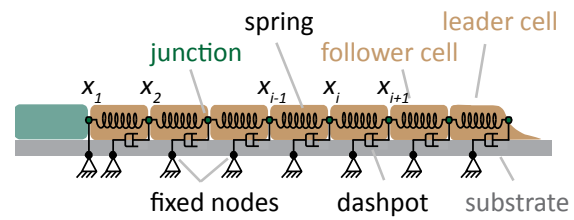

e

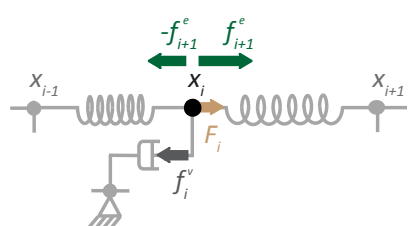

b

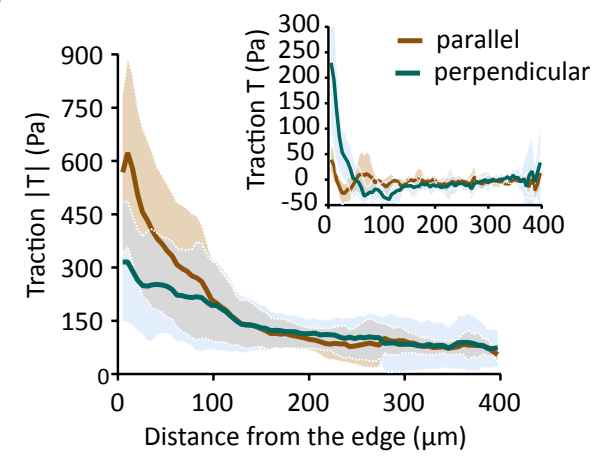

f

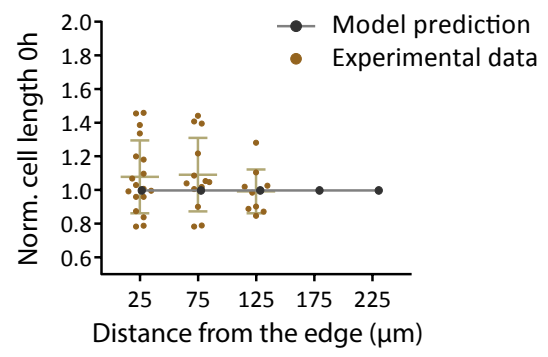

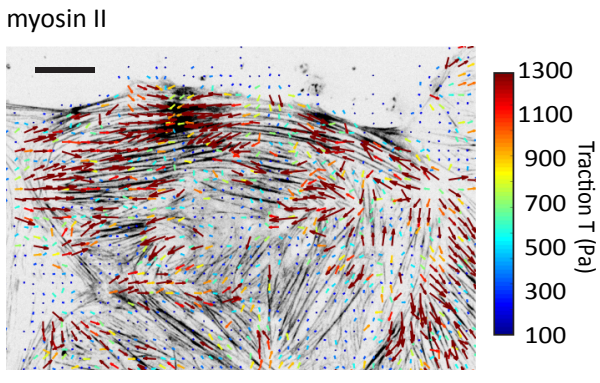

g

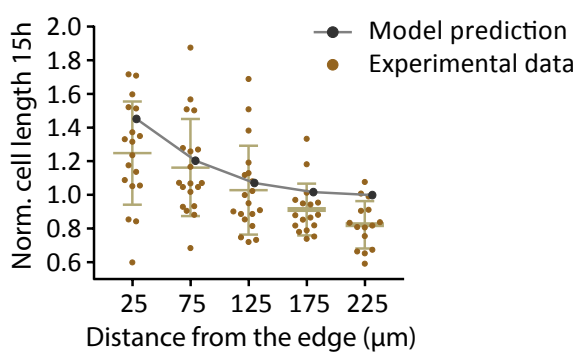


a

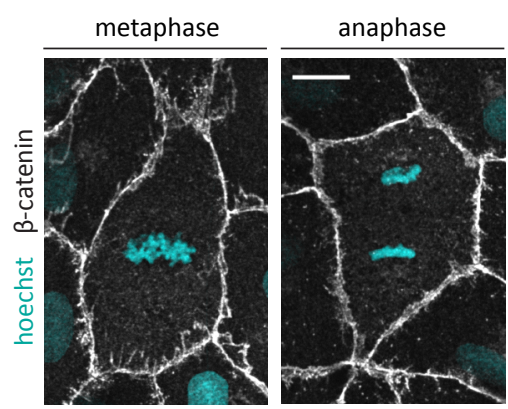

d

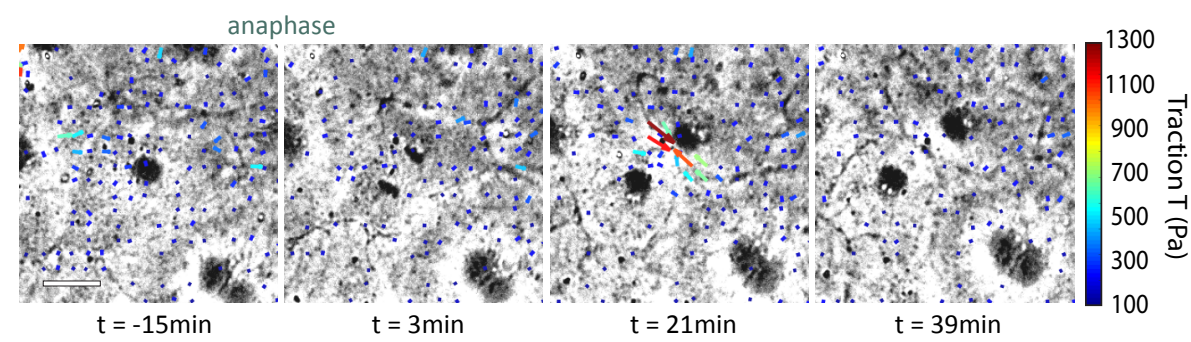

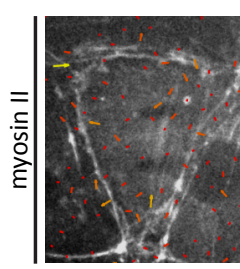

$\mathrm{t}=3 \min$

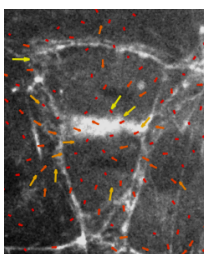

$\mathrm{t}=6 \mathrm{~min}$

cytokinetic ring

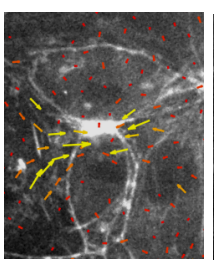

$t=15$ min

ring

h

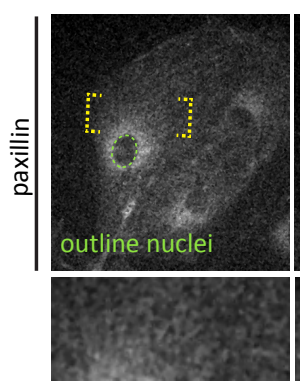

$\mathrm{t}=-30 \mathrm{~min}$

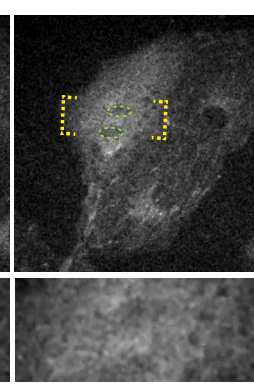

$\mathrm{t}=3 \min$

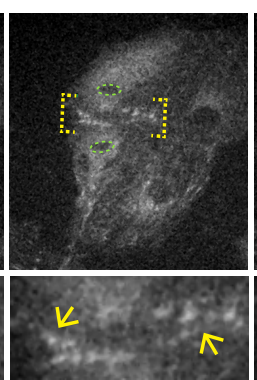

$\mathrm{t}=12 \mathrm{~min}$
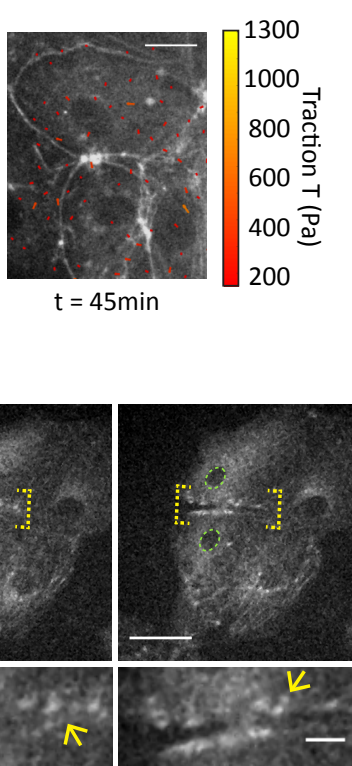

$\mathrm{t}=30 \mathrm{~min}$

\section{g}

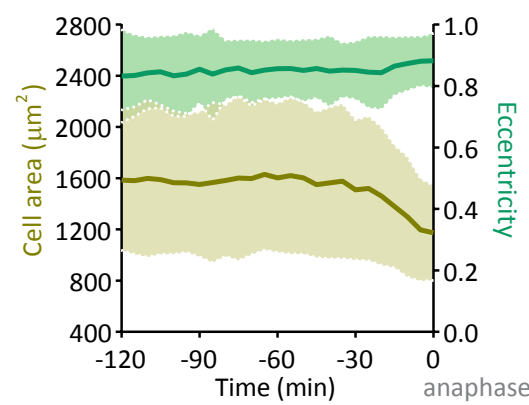

e
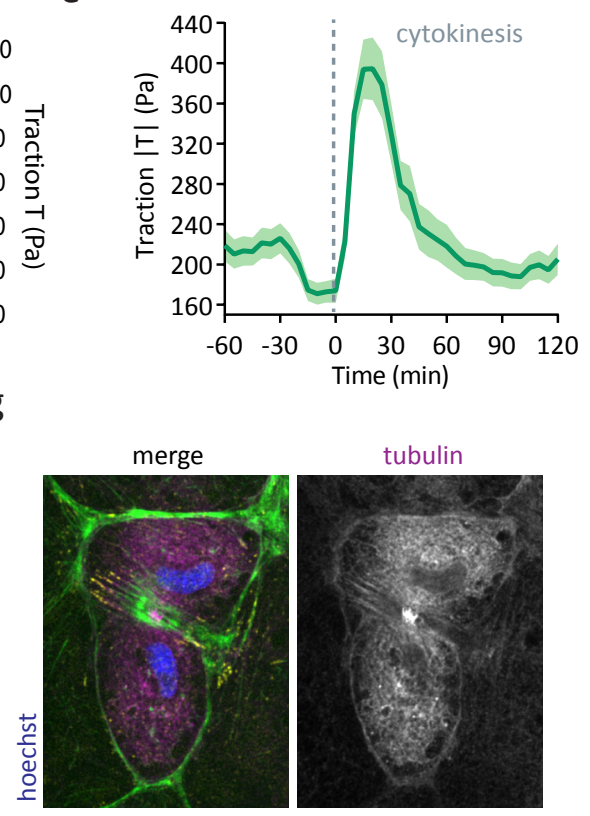

tubulin
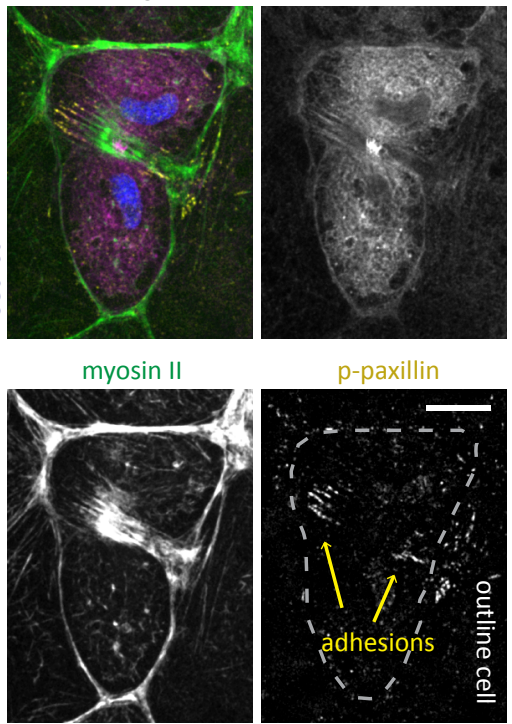

p-paxillin

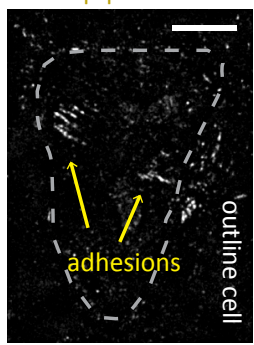

hoechst tubulin phalloidin
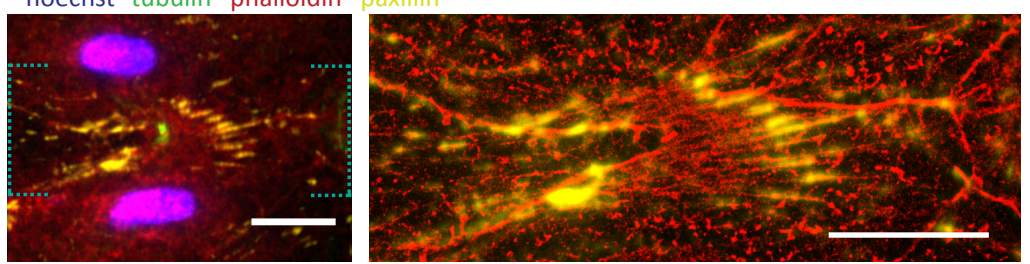

Figure 3 
a

$\beta$-catenin hoechst

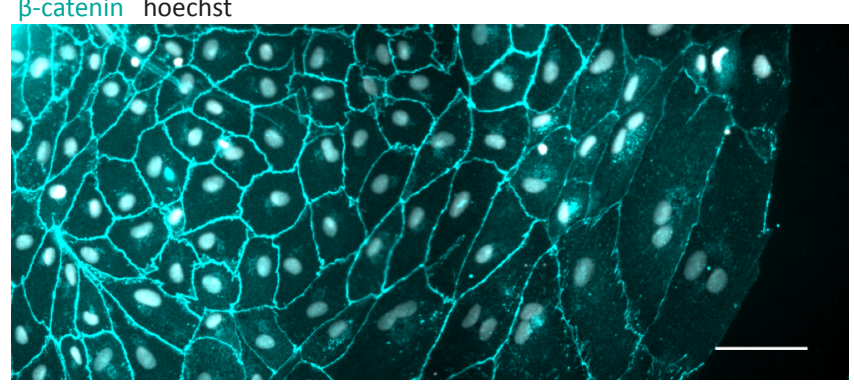

C

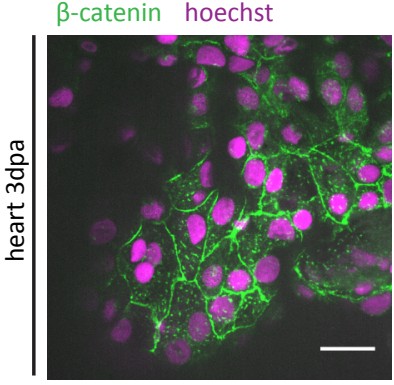

d b

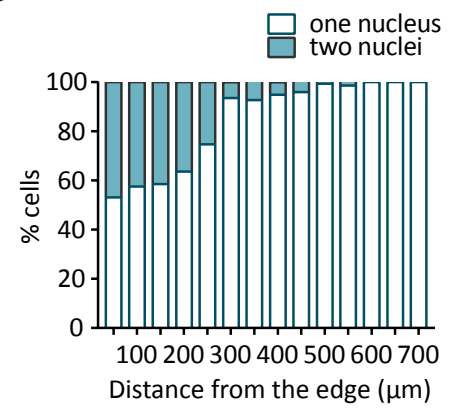

e

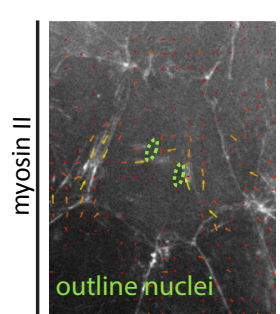

$t=3 \min$

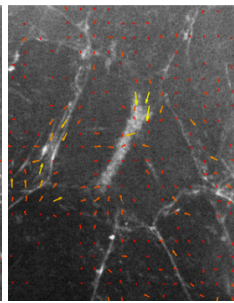

$t=6 \min$

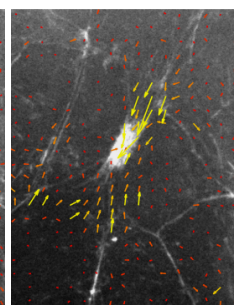

$\mathrm{t}=15 \mathrm{~min}$

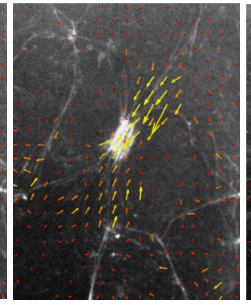

$\mathrm{t}=30 \mathrm{~min}$

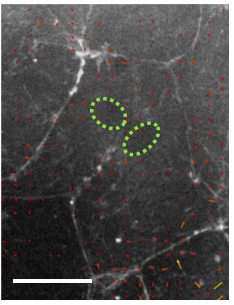

$t=108 \min$

hoechst

f

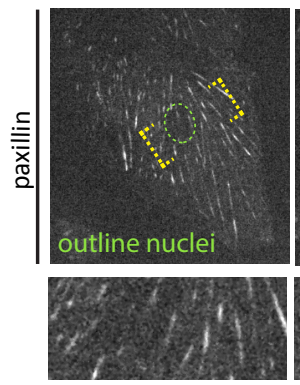

$t=-30 \mathrm{~min}$

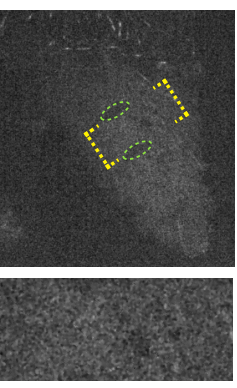

$\mathrm{t}=3 \mathrm{~min}$

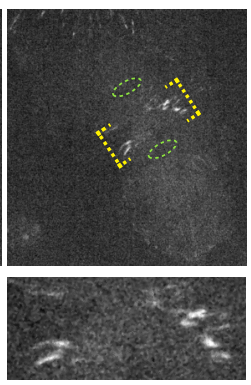

$t=12 \min$

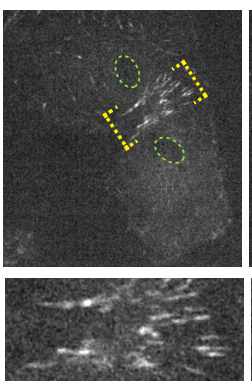

$\mathrm{t}=30 \mathrm{~min}$

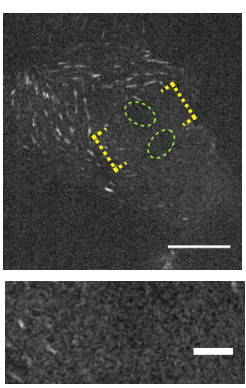

$\mathrm{t}=120 \mathrm{~min}$

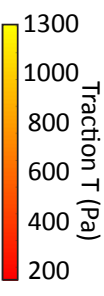


a

b
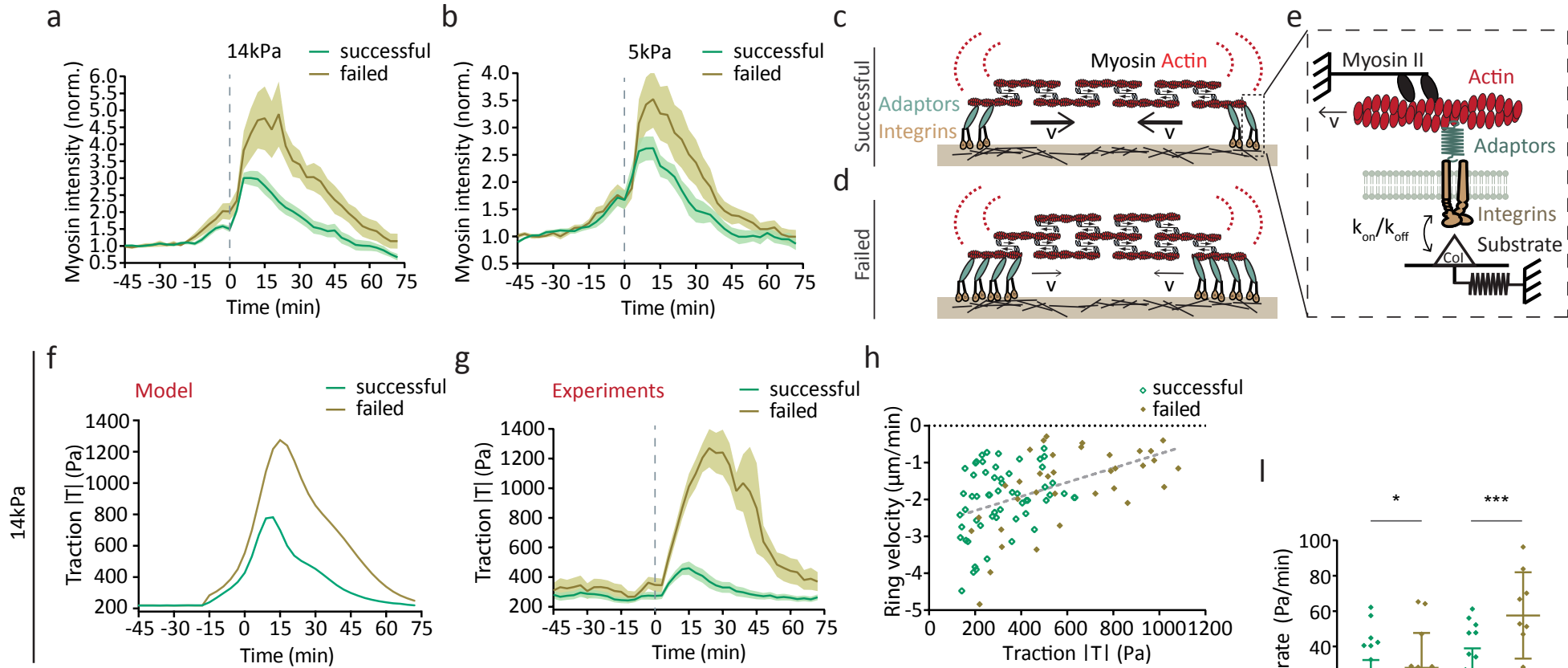

g

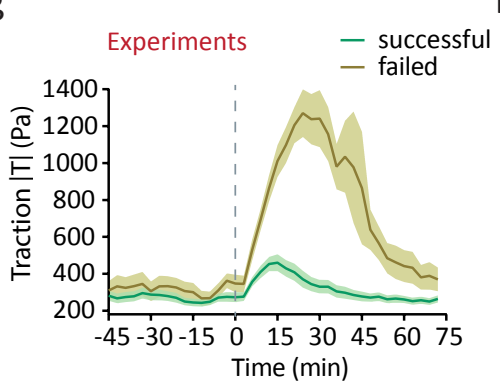

h
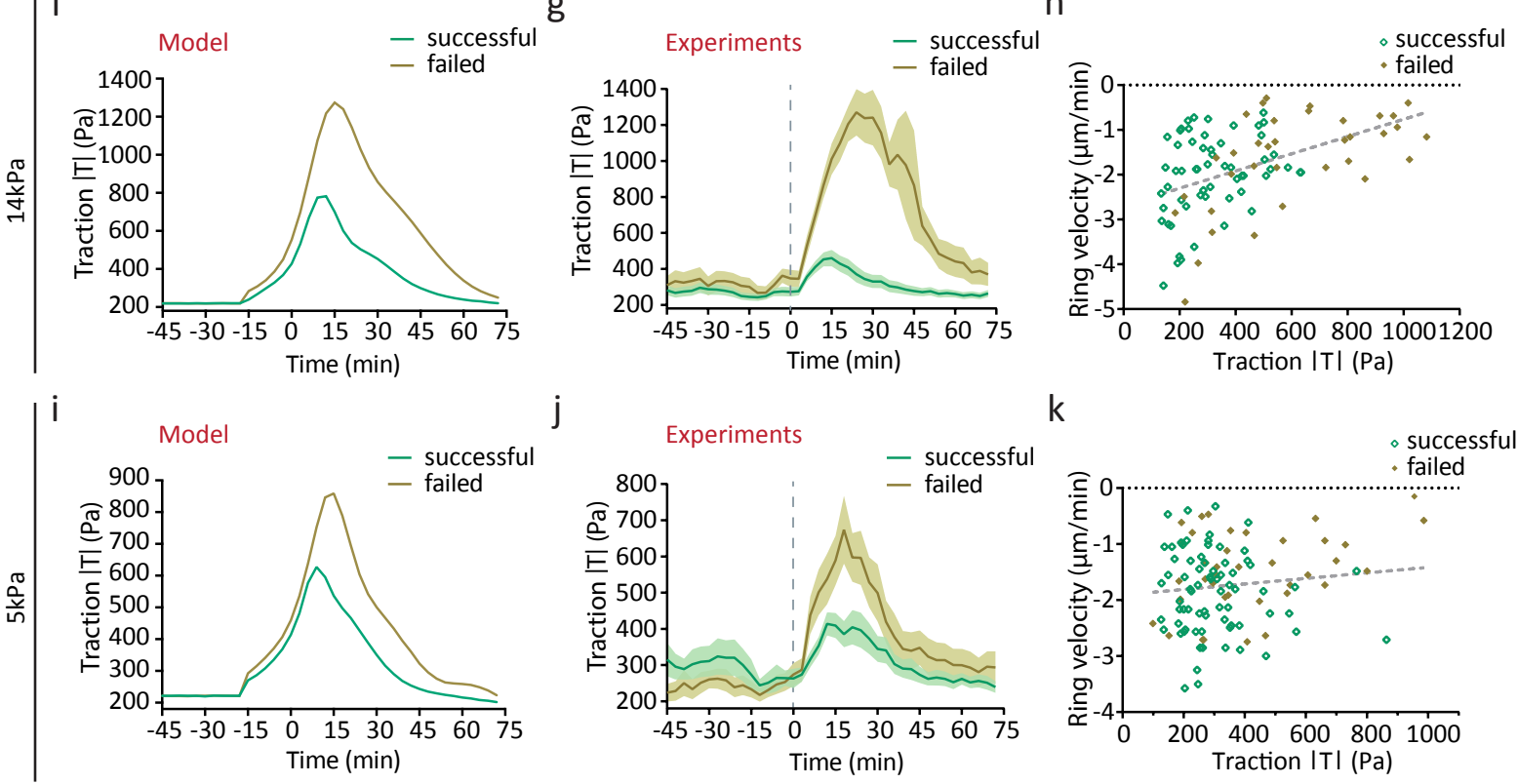

k

$\mathrm{m}$

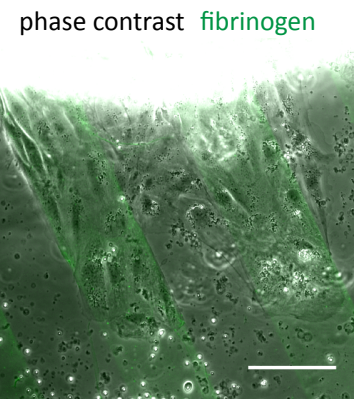

n

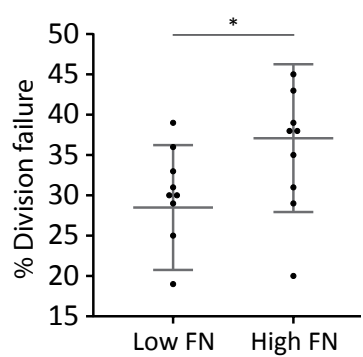

0

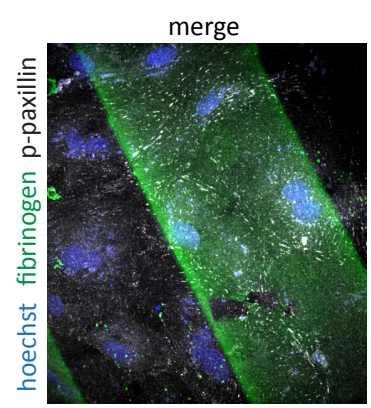

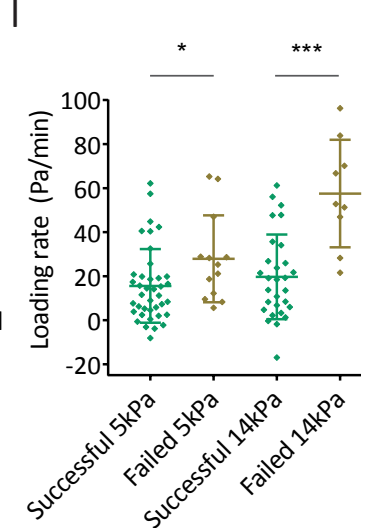

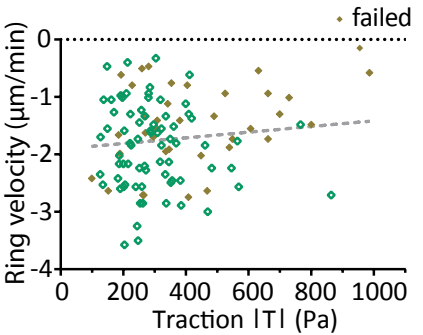




\title{
Supplementary Material
}

\author{
Marina Uroz ${ }^{1}$, Anna Garcia-Puig ${ }^{2,3}$, Isil Tekeli ${ }^{2,3}$, Alberto Elosegui-Artola ${ }^{1}$, Juan F. \\ Abenza $^{1}$, Ariadna Marín-Llauradó ${ }^{1}$, Silvia Pujals ${ }^{1}$, Vito Conte ${ }^{1,4}$, Lorenzo Albertazzi ${ }^{1,4}$, \\ Pere Roca-Cusachs ${ }^{1,5}$, Ángel Raya ${ }^{2,3,6}$, and Xavier Trepat ${ }^{1,3,5,6^{*}}$ \\ ${ }^{1}$ Institute for Bioengineering of Catalonia (IBEC), The Barcelona Institute for Science and Technology \\ (BIST), Barcelona, 08028, Spain \\ ${ }^{2}$ Center of Regenerative Medicine in Barcelona (CMRB), 08003 Barcelona, Spain \\ ${ }^{3}$ Centro de Investigación Biomédica en Red en Bioingeniería, Biomateriales y Nanomedicina (CIBER-BBN), \\ 08028 Barcelona, Spain \\ ${ }^{4}$ Department of Biomedical Engineering and the Institute for Complex Molecular Systems, Eindhoven \\ University of Technology, $5600 \mathrm{MB}$ Eindhoven, The Netherlands \\ ${ }^{5}$ University of Barcelona, 08028 Barcelona, Spain \\ ${ }^{6}$ Institució Catalana de Recerca i Estudis Avançats (ICREA), Barcelona, Spain. \\ * Corresponding author: xtrepat@ibecbarcelona.eu
}




\section{Supplementary Figures}

a
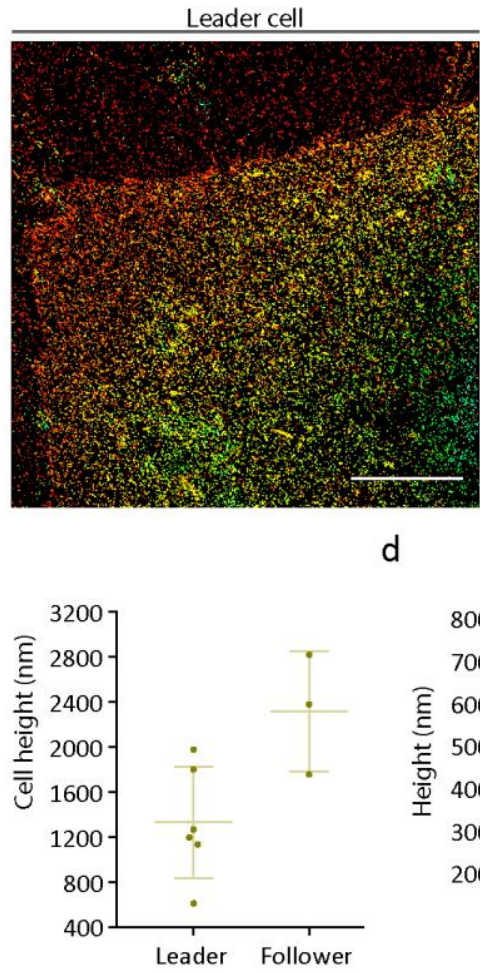

d

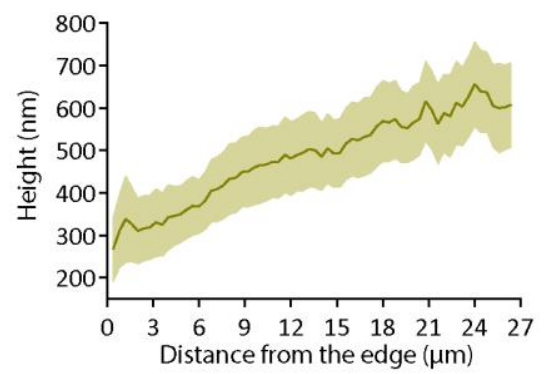

b

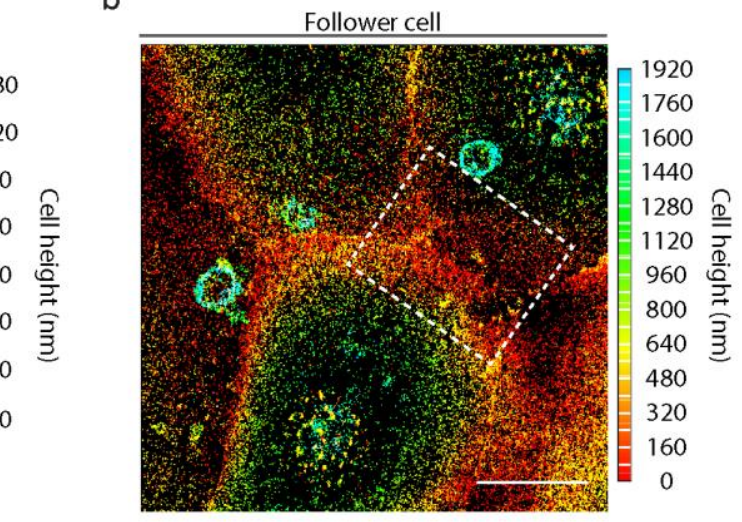

e

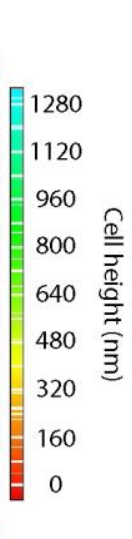

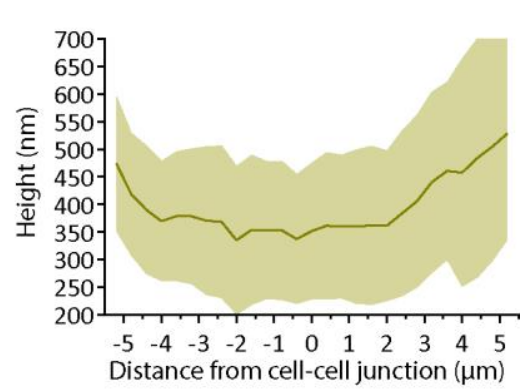

Supplementary Figure 1: The epicardium is squamous. (a) Representative leading edge cell. Color-coded cell height obtained from a STORM image of the cell membrane. Scale bar, $10 \mu \mathrm{m}$. (b) Representative follower cell. Color-coded cell height obtained from a STORM image of the cell membrane. Scale bar, $10 \mu \mathrm{m}$. (c) Mean maximum cell height for leading edge and follower cells ( $n=6$ leaders and 3 followers from 3 hearts). Error bars represent SD. (d) Mean membrane height as a function of the distance from the edge ( $n=6$ cells from 3 hearts). Shaded areas represent SEM. (e) Quantification of membrane height profile between two follower cells ( $\mathrm{n}=4$ cells from 3 hearts). Membrane height was averaged in a rectangular region containing a junction, illustrated by dashed white rectangle in (b). Averaging was performed along the direction parallel to the cell-cell junction. Shaded areas represent SEM. The image shown in a is representative of $n=6$ leader cells from 3 hearts and the image shown in $\mathbf{b}$ is representative of $n=3$ follower cells from 3 hearts. For all STORM measurements hearts were seeded on glass substrates. 
a

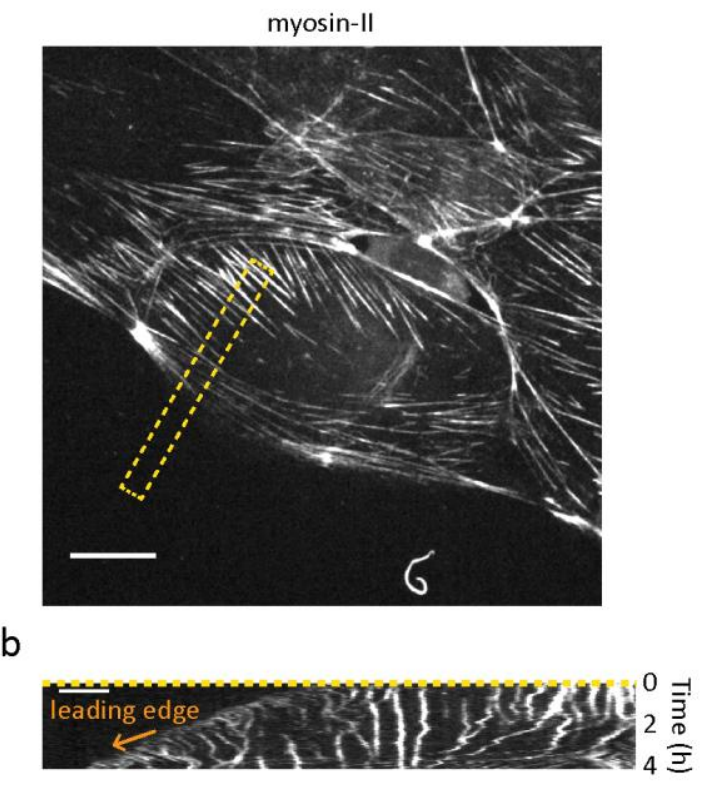

e

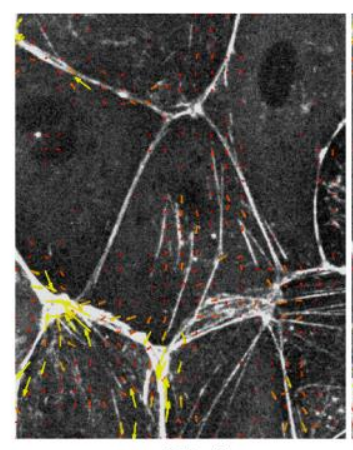

$\mathrm{t}=-30 \mathrm{~min}$
C

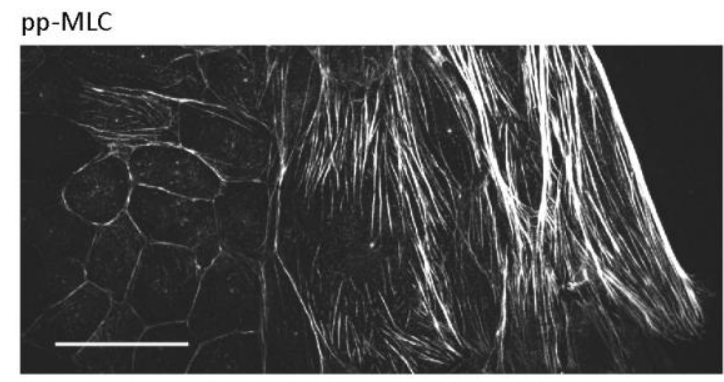

d

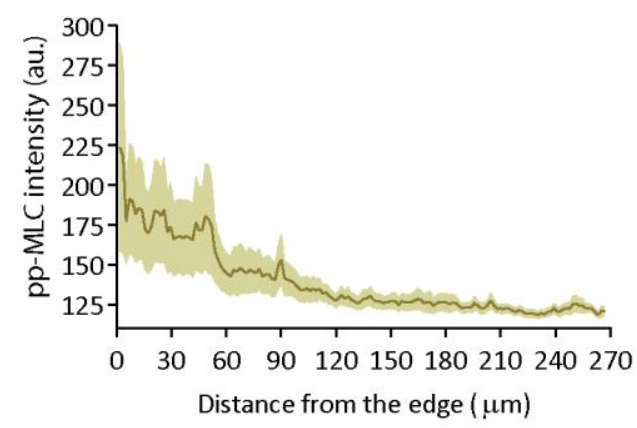

Supplementary Figure 2: Characterization of collective cell migration. (a) Snap shot of myosin-GFP at the leading edge of the explant. Scale bar, $20 \mu \mathrm{m}$. (b) Kymograph of myosin fluorescence intensity. Each row corresponds to an average of the yellow region marked on (a) (averaging is performed along the direction parallel to the short axis of the yellow rectangle, i.e., parallel to the leading edge). Scale bar, $5 \mu \mathrm{m}$. (c) Immunostaining of ppMLC in an epicardial monolayer. Scale bar, $50 \mu \mathrm{m}$. (d) Mean ppMLC intensity as a function of the distance from the edge ( $n=7$ monolayers from 3 hearts). ppMLC was averaged after subtracting the image background. Shaded areas represent SEM. (e) Tractions overlaid on myosin-GFP images in response to the spontaneous death of a follower cell. Surrounding cells quickly acquire a leader phenotype and migrate to cover the area left by the dead cell. The origin of the time axis (0min) is defined as the time point in which the follower cell spontaneously dies. Scale bar, $20 \mu \mathrm{m}$. Images shown in $\mathbf{a , b , \mathbf { e }}$ are representative of 6 hearts and the image shown in $\mathbf{c}$ is representative of 3 hearts. 


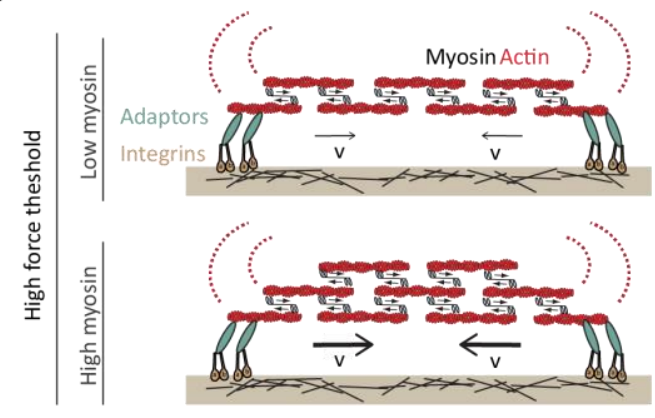

C

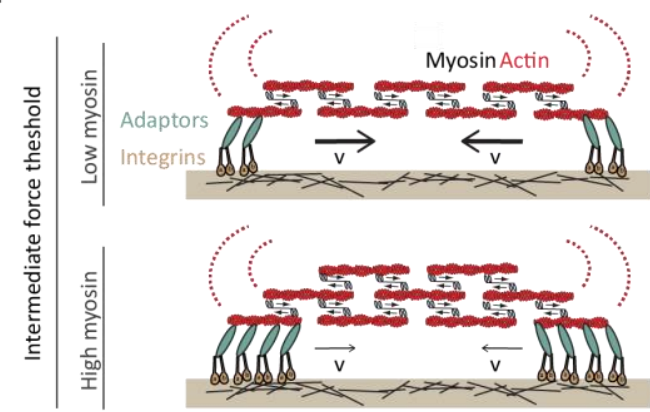

e

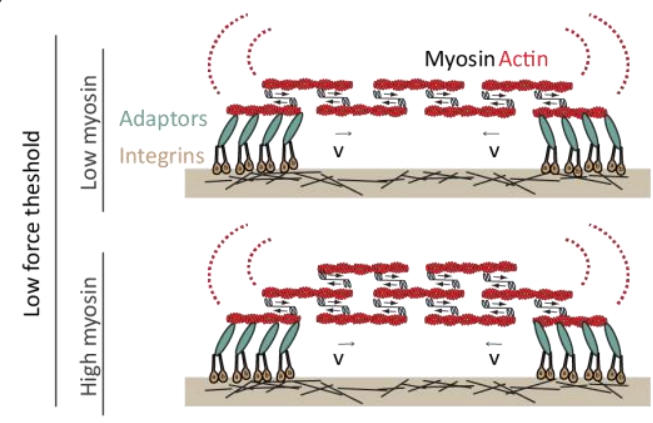

b

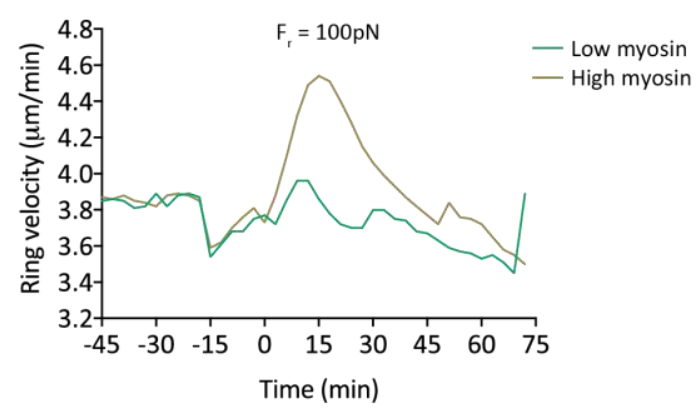

d

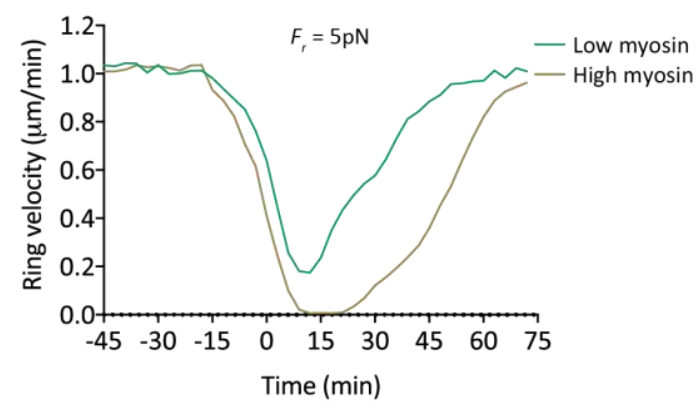

f

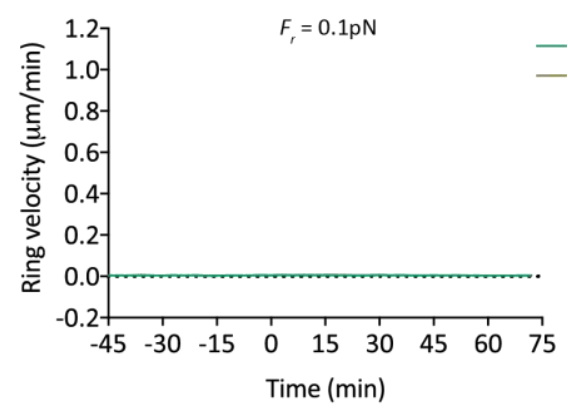

Supplementary Figure 3. Prediction of ring contraction velocities (actin flow) for different reinforcement thresholds. a-b For a high force reinforcement threshold (100 $\mathrm{pN}$ ), the ring containing higher myosin levels (bottom) closes faster than the ring with lower myosin levels (top). (a) Schematic of the cytokinetic ring adhered on the substrate. Top: low myosin concentration. Bottom: high myosin concentration. (b) Model prediction of actin flow velocity for the case of high and low myosin concentrations. c-d For an intermediate force reinforcement threshold $(5 \mathrm{pN})$ the ring with lower myosin levels (top) contracts faster than the ring with high myosin levels (bottom). (c) Schematic of the cytokinetic ring adhered on the substrate. (d) Model prediction of actin flow velocity for the case of high and low myosin concentrations. e-f For a low force reinforcement threshold $(0.1 \mathrm{pN})$ the rings become stuck to the substrate regardless of the relative myosin concentration. (e) Schematic of the cytokinetic ring adhered on the substrate. (f) Model prediction of actin flow velocity for the case of high and low myosin concentrations. For all simulations the myosin experimental values for $14 \mathrm{kPa}$ were used. 
a

Fn

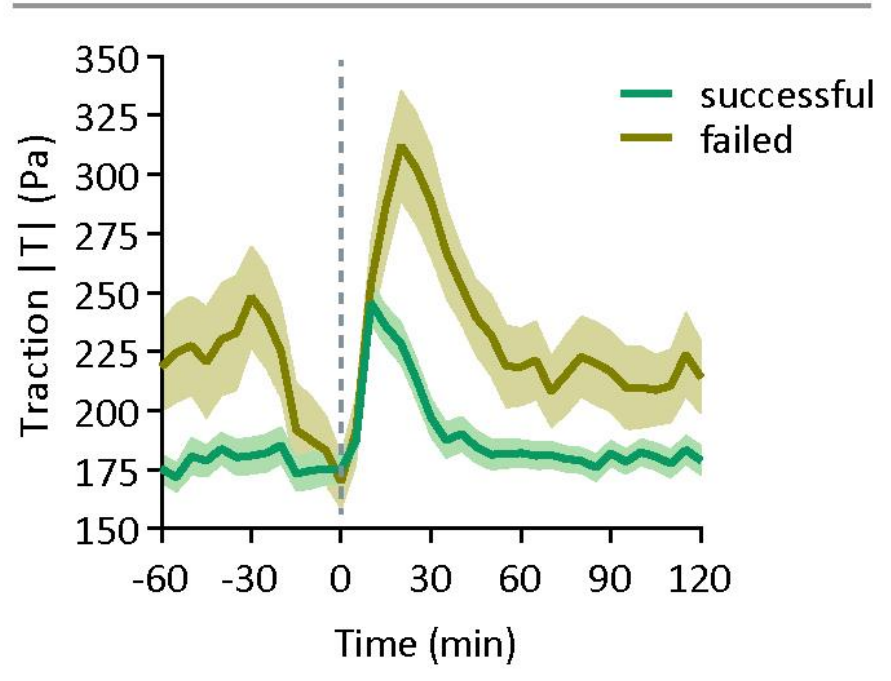

Supplementary Figure 4: Cytokinesis forces on fibronectin-coated gels. (a) Experimental time evolution of the traction force during division for successful and failed cytokinesis on $14 \mathrm{kPa}$ fibronectin-coated substrates. Dashed line represents the beginning of anaphase ( $\mathrm{n}=148$ successful and 35 failed divisions from 3 hearts). Tractions were averaged in a region of varying size enclosing the cytokinetic ring (illustrated in Supplementary Video 10). Shaded areas represent SEM. 


\section{Supplementary Videos}

Supplementary Video 1: Collective cell migration of a monolayer of epicardial cells explanting from the heart (left). Scale bar, $50 \mu \mathrm{m}$. Images are representative of 4 hearts.

Supplementary Video 2: Myosin dynamics during cell migration (cells expressing MyosinGFP). Right: cells at the leading edge. Left: follower cells. Scale bar, $20 \mu \mathrm{m}$. Images are representative of 6 hearts.

Supplementary Video 3: Myosin dynamics with tractions overlaid after a follower cell spontaneously dies. Scale bar, $20 \mu \mathrm{m}$. Images are representative of 6 hearts.

Supplementary Video 4: Phase contrast images of a monolayer of epicardial cells with overlaid tractions. Scale bar, $50 \mu \mathrm{m}$. Images are representative of 4 hearts.

Supplementary Video 5: Tractions during a successful division overlaid on phase contrast images. Scale bar, $10 \mu \mathrm{m}$. Images are representative of $\mathrm{n}=59$ divisions from 4 hearts.

Supplementary Video 6: Tractions during a successful division overlaid on myosin-GFP images. Scale bar, $10 \mu \mathrm{m}$. Images are representative of $n=29$ successful divisions from 4 hearts.

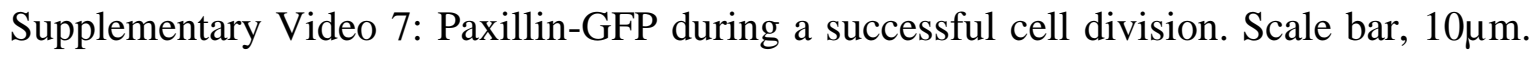
Images are representative of $n=13$ successful divisions from 2 hearts.

Supplementary Video 8: Tractions during a failed division overlaid on myosin-GFP images. Scale bar, $20 \mu \mathrm{m}$. Images are representative of $n=9$ failed divisions from 4 hearts.

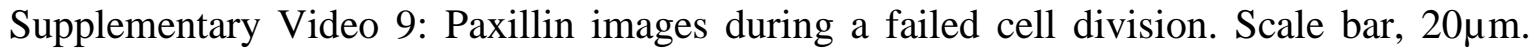
Images are representative of $n=5$ failed divisions from 2 hearts.

Supplementary Video 10: Calculation of average traction and myosin intensity. Average traction and myosin intensity at the ring were computed by averaging the corresponding signal in a region of interest of constant width $(13 \mu \mathrm{m}$ in Fig. 3 and $9 \mu \mathrm{m}$ in Fig. 5) and varying length (magenta outline) enclosing the cytokinetic ring. The length was determined by the cell shape in the direction parallel to the ring. The blue dashed line marks the cell outline. Myosin images are representative of $n=29$ successful divisions from 4 hearts. 


\section{$\underline{\text { Supplementary Tables }}$}

\begin{tabular}{|c|c|c|c|}
\hline Parameter & Meaning & Value & Origin \\
\hline$n_{m 0}$ & $\begin{array}{l}\text { Initial number of myosin } \\
\text { motors }\end{array}$ & 200 & Adjusted \\
\hline$n_{c l}$ & $\begin{array}{c}\text { Number of collagen } \\
\text { molecules }\end{array}$ & 70 & Adjusted \\
\hline$k_{\text {ont }}$ & True binding rate & $2.5^{*} 10^{-4} \mu \mathrm{m}^{2} / \mathrm{s}$ & $\begin{array}{c}\text { Adjusted, of the order of } \\
\text { values reported for } \alpha \operatorname{IIB} \beta 3\end{array}$ \\
\hline$d_{\text {clu }}$ & Density of clutches & 50 & Adjusted \\
\hline$k_{\text {off }}$ & $\begin{array}{l}\text { Unbinding rate of } \\
\text { integrins from collagen }\end{array}$ & 0.2 (catch bond) & $\begin{array}{c}\text { Adjusted, of the order of other } \\
\text { integrins } 43,74\end{array}$ \\
\hline$F_{r}$ & Reinforcement Force & $5 \mathrm{pN}$ & Set \\
\hline$v_{u}$ & $\begin{array}{l}\text { Unloading myosin motor } \\
\text { velocity }\end{array}$ & $110 \mathrm{~nm} / \mathrm{s}$ & 46,48 \\
\hline$F_{m}$ & Myosin motor stall force & $2 \mathrm{pN}$ & 75 \\
\hline$k_{\text {clu }}$ & Clutch spring constant & $1 \mathrm{nN} / \mathrm{nm}$ & 76 \\
\hline$d_{\text {add }}$ & $\begin{array}{l}\text { Integrins added after each } \\
\text { reinforcement event }\end{array}$ & $24 / \mu \mathrm{m}^{2}$ & Does not affect model output \\
\hline$r_{a}$ & Radius of adhesion & $700 \mathrm{~nm}$ & Set \\
\hline
\end{tabular}

Supplementary Table 1. Clutch model parameters 